\title{
Progression of a Muscular Dystrophy Due to a Genetic Defect in Membrane Synthesis is Driven by Large Changes in Neutral Lipid Metabolism
}

Mahtab Tavasoli

Dalhousie University https://orcid.org/0000-0001-7962-3566

Sarah Lahire

University of Reims Champagne-Ardenne

Stanislav Sokolenko

Dalhousie University

J. Pedro Fernández-Murray1

Dalhousie University

Kitipong Uaesoontrachoon

Dalhousie University/Agada Biosciences

Abir Lefsay

Dalhousie University

Joyce Rowsell

Agada Biosciences

Sadish Srinivassane

Agada Biosciences

Molly Praest

Agada Biosciences

Alexandra MacKinnon

Agada Biosciences

Melissa Mammoliti

Agada Biosciences

Ashley Maloney

Agada Biosciences

Marina Moraca

Agada Biosciences

Kanneboyina Nagaraju

Department of Pharmaceutical Sciences, School of Pharmacy and Pharmaceutical Sciences, Binghamton University

Eric Hoffman

School of Pharmacy and Pharmaceutical Sciences 
Christopher McMaster ( $\square$ christopher.mcmaster@dal.ca )

Dalhousie University

\section{Article}

Keywords: metabolism, muscular dystrophy, skeletal muscle, lipid, acylcarnitine, triacylglycerol, phosphatidylcholine, peroxisome proliferator-activated receptors (PPAR)

Posted Date: May 19th, 2021

DOI: https://doi.org/10.21203/rs.3.rs-64129/v1

License: (c) (i) This work is licensed under a Creative Commons Attribution 4.0 International License. Read Full License

Version of Record: A version of this preprint was published at Nature Communications on March 23rd, 2022. See the published version at https://doi.org/10.1038/s41467-022-29270-z. 


\section{Progression of a Muscular Dystrophy Due to a Genetic Defect in Membrane Synthesis is Driven by Large Changes in Neutral Lipid Metabolism}

Mahtab Tavasoli ${ }^{1}$, Sarah Lahire ${ }^{2}$, Stanislav Sokolenko ${ }^{3}$, J. Pedro Fernandez-Murray ${ }^{1}$, Kitipong Uaesoontrachoon ${ }^{4}$, Abir Lefsay ${ }^{5}$, Joyce Rowsell ${ }^{4}$, Sadish Srinivassane ${ }^{4}$, Molly Praest $^{4}$, Alexandra MacKinnon ${ }^{4}$, Melissa Stella Mammoliti ${ }^{4}$, Ashley Alyssa Maloney ${ }^{4}$, Marina Moraca ${ }^{4}$, Kanneboyina Nagaraju ${ }^{4,6}$, Eric P. Hoffman ${ }^{4,6}$, and Christopher R. McMaster $^{1}$

${ }^{1}$ Department of Pharmacology, Dalhousie University, Halifax, Nova Scotia, Canada ${ }^{2}$ University of Reims Champagne-Ardenne, Reims, France

${ }^{3}$ Department of Process Engineering \& Applied Science, Dalhousie University, Halifax, Nova Scotia, Canada

${ }^{4}$ Agada Biosciences Inc., Halifax, Nova Scotia, Canada

${ }^{5}$ Mass Spectrometry Core Facility, Dalhousie University, Halifax, Nova Scotia, Canada

${ }^{6}$ School of Pharmacy and Pharmaceutical Sciences, Binghamton University, State University of New York (SUNY), Binghamton, NY, USA

\section{Correspondence}

christopher.mcmaster@dal.ca 


\section{Abstract}

$C H K B$ encodes one of two mammalian choline kinase enzymes that catalyze the first step in the synthesis of the major membrane phospholipid, phosphatidylcholine (PC). In humans, inactivation of the CHKB gene causes a recessive form of a rostral-to-caudal congenital muscular dystrophy. Using Chkb knockout mice, we reveal that at no stage of the disease is PC level significantly altered. Instead, at early stages of the disease the level of mitochondrial specific lipids acylcarnitine (AcCa) and cardiolipin (CL) increase 15-fold and 10-fold, respectively. Importantly, these changes are only observed in affected muscle and contribute to the decrease in the skeletal muscle functional output in these mice. As the disease progresses, AcCa and CL levels normalize and there is a 12-fold increase in the neutral storage lipid triacylgycerol and a 3-fold increase in its upstream lipid diacylglycerol. Our findings indicate that the major changes in lipid metabolism upon loss of function of Chkb is not a change in PC level, but instead is an initial inability to utilize fatty acids for energy resulting in shunting of fatty acids into triacyglycerol.

Keywords - metabolism, muscular dystrophy, skeletal muscle, lipid, acylcarnitine, triacylglycerol, phosphatidylcholine, peroxisome proliferator-activated receptors (PPAR) 


\section{Introduction}

Phosphatidylcholine (PC) is the major phospholipid present in mammalian cells, comprising approximately $50 \%$ of phospholipid mass. Choline kinase catalyzes the phosphorylation of choline to phosphocholine and is the first enzymatic step in the synthesis of PC ${ }^{1}$. There are two genes that encode human choline kinase enzymes, CHKA and CHKB. Monomeric choline kinase proteins combine to form homo- or heterodimeric active forms ${ }^{2}$. CHKA and CHKB proteins share similar structures and enzyme activity but display some distinct molecular structural domains and differential tissue expression patterns. Knock-out of the murine Chka gene leads to embryonic lethality ${ }^{3}$. Chkb deficient $\left(\mathrm{Chkb}^{--}\right)$mice are viable, but noticeably smaller than their wild type counterparts, and show severe bowing of the ulna and radius at birth. By 2-3 months of age $\mathrm{Chkb}^{-/}$mice lose hindlimb motor control, while the forelimbs are spared ${ }^{4,5}$. Inactivation of the Chkb gene in mice would be predicted to decrease PC level, however, reports indicate no, or a very modest, decrease in PC level in Chkb-/ mice, and this decrease is similar in both forelimb and hindlimb muscle ${ }^{6,7}$. The very small decrease in PC mass, and the fact that there is no rostral-to-caudal change in PC, suggest a poor correlation of the anticipated biochemical defects and observed rostralto-caudal phenotype of this muscular dystrophy ${ }^{5}$. It is unclear how a defect in a gene required for the synthesis the major phospholipid in mammalian cells causes a muscular dystrophy, especially in light of the fact that global inactivation of the $C H K B / C h k b$ gene (human or mouse) does not affect the level of the product of its biochemical pathway, PC. 
Muscular dystrophy, congenital, megaconial type (OMIM 602541) is an autosomal recessive dystrophy caused by loss of function of $C H K B$ gene, and is the only defect in phospholipid synthesis that can cause a muscular dystrophy ${ }^{5,8-14}$. Muscular dystrophies have been mapped to at least 30 different causal genes ${ }^{15}$. The most common types of muscular dystrophy result from mutations in genes coding for members of protein complexes which act as linkers between the cytoskeleton of the muscle cell and the extracellular matrix that provides mechanical support to the plasma membrane during myofiber contraction ${ }^{16,17}$. Muscular dystrophies result in fibrofatty replacement of muscle tissue, progressive muscle weakness, functional disability and often early death 18 19,20.

Skeletal muscle accounts for $20-30 \%$ of whole body basal metabolic rate ${ }^{21}$. Fatty acid oxidation is the major source of ATP for skeletal muscle during the resting state ${ }^{22}$. Fatty acids can be synthesized de novo by cells or can be obtained extracellularly, with the bulk of lipids delivered to cells through the circulation via serum albumin or lipoprotein/lipoprotein receptors. For fatty acids to be metabolized they are first activated by esterification to fatty acyl-CoA. Subsequently, they have divergent fates depending on the metabolic status of the cells (Fig. 1). The three major fates of fatty acids are 1. conversion to fatty acyl carnitine for subsequent mitochondrial $\beta$-oxidation to provide energy, 2. the synthesis of neutral lipid species for storage as triacylglycerol (TG) rich cytoplasmic lipid droplets, or 3. metabolism into phospholipids, such as PC, to maintain membrane integrity. Fatty acids can also directly bind peroxisome proliferatoractivated receptors (PPARs), key players in the regulation of lipid metabolism by altering the expression of genes required for the conversion of fatty acids to fatty acyl- 
CoA for phospholipid and TG synthesis, and for fatty acid activation to acylcarnitine (AcCa) for entry into mitochondria and subsequent fatty acid $\beta$-oxidation ${ }^{23}$.

In the present study, we use mouse and cell models to investigate the temporal changes in lipid metabolism in the absence of the Chkb gene. Results demonstrate that PC level remains essentially unchanged. Instead, this genetic defect in PC synthesis drives large fluctuations in mitochondrial lipid metabolism with an inability to use fatty acids for mitochondrial $\beta$-oxidation resulting in a temporal shunting of fatty acids into TG and their storage as lipid droplets. These changes were specific to affected muscle. This study provides insight into the surprising biochemical phenotype whereby a genetic block in a lipid metabolic pathway does not directly affect the product of its pathway, and instead alters tangential pathways in a manner that explains the rostral-to-caudal gradient of a genetic disease.

\section{Results}

\section{Choline kinase deficient mice display hallmark muscular dystrophy phenotypes}

To address the extent that mice lacking Chkb function display gross muscular dystrophy phenotypes, we tested muscle function in $\mathrm{Chkb}^{+/+}, \mathrm{Chkb}^{+/}$and $\mathrm{Chkb}^{-/-}$mice from 6 weeks to 20 weeks of age using a grip strength assay and a total distance run to exhaustion test. Body weight was also recorded each week at similar times over the duration of the phenotyping experiments. Body weight of the $\mathrm{Chkb}^{+/+}$and $\mathrm{Chkb} \mathrm{b}^{+/}$mice showed no difference between groups (Fig. 2a). The Chkb-/- mice weighed significantly less than their wild type counterparts at all time points. The average body weight of 
$\mathrm{Chkb}^{-/}$mice was $33 \%$ to $42 \%$ less than that of $\mathrm{Chkb}^{+/+}$mice at week 6 and week 20 , respectively.

Forelimb grip strength measurements were performed at three different timepoints and normalized to body weight. The $\mathrm{Chkb}^{-/}$mice had significantly lower (less than half) the normalized forelimb strength than wild type mice at all three timepoints (week 6, 12 and 18) (Fig. 2b). Another measure of neuromuscular function is the resistance to treadmill running, evaluated as the total distance that each mouse is able to run until exhaustion. The test was performed in all groups at three timepoints (Week 7, 13 and 19). The total distance covered by the wild type mice before exhaustion was similar at all 3 time points (Fig. 2c). There was no significant difference between $\mathrm{Chkb}^{+/+}$ and $\mathrm{Chkb}^{+/}$groups, these mice maintained the ability to cover the same total distance before exhaustion (week 7 vs. week 19; non-significant). At week 7 , the $C h k b^{-/}$mice showed a basal level of total distance run that was $50 \%$ that of the wild type or $C h k b^{+/-}$ mice. Moreover, the $\mathrm{Chkb}^{-/}$mice showed a decline in running performance from week 7 to week 19 , with an almost complete inability to run observed by week 19 . Gross measurements of neuromuscular strength in whole mice demonstrate that mice heterozygous for Chkb gene display similar phenotypes to wild type mice. Notably, mice lacking both copies of the Chkb gene display a significant decrease in overt neuromuscular phenotypes.

The level of circulating creatinine kinase (CK), a biomarker of sarcolemmal injury, was determined in $\mathrm{Chkb}^{+/+}, \mathrm{Chkb}^{+/}$, and $\mathrm{Chkb}^{-/}$mice. No significant change in the serum level of $\mathrm{CK}$ was observed in $\mathrm{Chkb}^{+/}$heterozygous mice when compared to the 
wild type. CK activity was 2.5 -fold higher in $\mathrm{Chkb}^{-/}$null mice than that of wild type mice (Fig. 2d).

To determine if the decreased neuromuscular phenotypes observed in the $\mathrm{Chkb}^{-/-}$ mice were due to a direct effect on muscle itself, maximal specific force generated by freshly isolated extensor digitorum longus (EDL) muscle from the hindlimb of $\mathrm{Chkb}^{+/+}$, $\mathrm{Chkb}^{+/}$, and $\mathrm{Chkb}^{-/-}$mice at week 20 was determined. EDL muscle fatigue was measured with 60 isometric contractions for $300 \mathrm{~ms}$ each, once every $5 \mathrm{sec}$, at $250 \mathrm{~Hz}$. There was no significant difference between wild type and heterozygous Chkb mice in regard to specific force decrease during fatigue and specific force generation, (Fig. 2e, f). $\mathrm{Chkb}^{-/-}$mice displayed a specific EDL force that was $10 \%$ that of $\mathrm{Chkb}^{+/+}$or $\mathrm{Chkb}^{+/-}$ mice. In addition, $\mathrm{Chkb}^{-/}$mice were at maximally fatigued levels, that is those observed in $\mathrm{Chkb}^{+/+}$or $\mathrm{Chkb}^{+/-}$mice after 60 muscle stimulations, at the first stimulation. Hindlimb muscle from $\mathrm{Chkb}^{-/}$mice produce less force, and are much more easily fatigued, than that of wild type or Chkb heterozygous mice.

Similar to humans ${ }^{8,10}$, mice with one functional copy of the CHKB gene do not possess any obvious overt muscle dysfunction, whereas mice that are homozygous null for functional copies of the Chkb gene display hallmark muscular dystrophy phenotypes.

Chka protein expression is inversely correlated with the rostro-caudal gradient of severity in Chkb-mediated muscular dystrophy

Consistent with the rostral-to-caudal nature of Chkb associated muscular dystrophy, transmission electron micrographs of 115 day old $\mathrm{Chkb}^{-/}$mice show extensive injury in hindlimb (quadriceps and gastrocnemius) but not the forelimb (triceps) (Supplementary 
Fig. 1a-c). Chkb encodes choline kinase b, the first enzymatic step in the synthesis of PC, the most abundant phospholipid present in eukaryotic membranes. A second choline kinase, Chka is present in mouse (and human) tissues. We investigated whether the lack of dystrophic phenotypes in $\mathrm{Chkb}^{+/-}$mice, and the rostro-caudal gradient of muscular dystrophy in $\mathrm{Chkb}^{-/}$muscle, can be explained by compensatory changes in Chkb or Chka protein levels using western blot. In Chkb/- mice, there was a $\sim 50 \%$ decrease in Chkb protein detected in both the forelimb and hindlimb muscles of Chkb ${ }^{+/-}$mice compared to wild type (Fig. 3a, b). There was no change in Chka protein level in hindlimb muscle of $\mathrm{Chkb}^{+/-}$mice compared to wild type, and a small but statistically insignificant increase in Chka level in forelimb muscle.

In $\mathrm{Chkb}^{-/-}$mouse forelimb or hindlimb muscle, Chkb protein expression was undetectable consistent with the allele not producing Chkb protein. In forelimb muscle from $\mathrm{Chkb}^{-/}$mice there was a compensatory upregulation of Chka protein expression to almost 3-fold that observed in wild type mice. In contrast, in hindlimb muscle from Chkb/- mice Chka protein expression was decreased to less than $10 \%$ that observed in wild type mice. A compensatory level of Chka protein expression inversely correlates with the rostro-caudal gradient of severity in $\mathrm{Chkb}^{-/-}$associated muscular dystrophy.

\section{Loss of Chkb activity exerts a major effect on neutral lipid abundance}

PC synthesis is integrated with the synthesis of other major phospholipid classes, as well as AcCa, fatty acids and the neutral lipids diacylglycerol and triacylglycerol (Fig.1). Lipidomics was used to determine if complete loss of Chkb function, and the associated upregulation of Chka in the forelimb but not hindlimb muscle of $\mathrm{Chkb}^{-/-}$mice, 
differentially altered lipid metabolism. The levels of the major glycerophospholipids, neutral lipids and acylcarnitine in hindlimb and forelimb muscle isolated from 12 day old

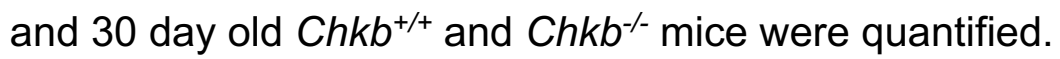

In the forelimb and hindlimb muscle of both 12 day old and 30 day old Chkbmice, the level of PC was the same as wild type mice (Fig. 4a-d). In 12 day old Chkb-/mice the largest change observed was a 15-fold increase in AcCa level in hindlimb muscle, and to a lesser degree ( 2-fold increase) in forelimb, compared to their wild type littermates. The second largest change in 12 day old mice was a 10 -fold increase in the level of cardiolipin $(\mathrm{CL})$ in hindlimb muscle that was not present in forelimb muscle of $\mathrm{Chkb}^{-/}$mice. Phosphatidylethanolamine (PE) and phosphatidylinositol (PI) levels were also slightly increased ( 1.5 fold) in both forelimb and hindlimb muscles of 12 day old $\mathrm{Chkb}^{-/}$mice. The large changes in lipid levels in hindlimb muscle, versus forelimb, of $\mathrm{Chkb}^{-/}$mice are consistent with the rostral-to-caudal nature of the muscular dystrophy observed in these mice.

Considering the progressive nature of the disease, we tracked the changes in the lipid profile in the hindlimb of 30 day old $C h k b^{-/}$mice, when muscle injury is more pronounced. In sharp contrast to 12 day old mice, AcCa and CL levels were no longer increased and were at the same level as wild type mice. Instead, there was a 12-fold increase in the neutral storage lipid TG and a 3-fold increase in its precursor DG in the hindlimb samples of $\mathrm{Chkb}^{-/}$mice (Fig. 4e, f). PE and PS levels were 2-3-fold higher in the hindlimb samples from 30 day old $\mathrm{Chkb}^{-/}$mice compared to wild type littermates. There is a temporal shift from a 12 to 15 fold increase in $\mathrm{CL}$ and $\mathrm{AcCa}$, to a similar increase in TG, only in affected muscle in $C h k b^{-/}$mice. 
As AcCa levels are many fold higher than wild type mice in the early stage of $\mathrm{Chkb}^{-/}$muscular dystrophy, this implies that the affected muscles are defective in using fatty acids for the production of cellular energy by mitochondrial $\beta$-oxidation. As $C h k^{-1-}$ muscular dystrophy progresses, the affected muscles appear to adapt to this inability to consume fatty acids by transitioning toward energy storage indicated by the large increase in TG.

\section{Increased intramyocellular lipid droplet accumulation and enlarged mitochondria in hindlimb muscles from Chkb ${ }^{-/}$mice}

To understand early ultrastructural pathological changes, and to further explore the nature of the accumulation of TG in affected muscle as $\mathrm{Chkb}^{-/}$muscular dystrophy progresses, we performed transmission electron microscopy (TEM) on hindlimb muscles from 12 and 115 day old mice. Consistent with previous reports ${ }^{5}$, our transmission electron images of $\mathrm{Chkb}^{-/}$mice showed that the muscular dystrophy in forelimb is extremely mild compared to the hindlimb (Supplementary Fig. 1a), while both limbs do display bone abnormalities ${ }^{24}$. A closer examination of hindlimb muscle TEM images from 12 day old mice revealed early signs of disrupted sarcomeres, as well as a small increase in the abundance of cytoplasmic lipid droplets, consistent with the small (2-fold) but statistically not significant increase in TG in hindlimb muscle we observed using lipidomics. Interestingly, these lipid droplets were located mainly adjacent to enlarged mitochondria (Fig. 5a). Detailed quantification of randomly imaged lipid droplets in hindlimb muscle from 12 day old $\mathrm{Chkb}^{-/}$mice determined that $81 \%$ were 
associated with mitochondria (Fig. 5a and Supplementary Fig. 2c). In 115 day old Chkb- mice, cytoplasmic lipid droplets increased substantially in size (Fig. 5a).

We also evaluated TG accumulation in muscle using confocal microscopy by staining hindlimb muscle sections of 30 day old $\mathrm{Chkb}^{-/}$mice with BODIPY 493/503 (Fig. $5 b)$. Concanavalin A dye conjugate ( $\mathrm{CF}^{\mathrm{TM}}$ 633) and Dapi were used to stain membrane (Red) and nucleus (Blue) respectively. Consistent with our TEM and lipidomics results, BODIPY-stained lipid droplets were noticeably more frequent and larger in $\mathrm{Chkb}^{-/-}$ hindlimb muscles compared to the wild type littermates. The same pattern of lipid droplet staining was observed using Nile red staining (Supplementary Fig. 2a, b).

The lipidomics results point to large changes in mitochondrial specific lipids at the early stages of Chkb associated muscular dystrophy. To further explore the nature of these changes, we investigated the temporal development of morphological changes in mitochondria in hindlimb muscle of $\mathrm{Chkb}^{-/-}$mice using standard TEM stereological methods ${ }^{25}$. The results show that at 12 days of age, the size of mitochondria increased $\left(6.2 \% \pm 0.5\right.$ vs $11.4 \% \pm 1.6 ; P<0.01$; wild type vs $\left.C h k^{-/-}\right)$while the number of mitochondria (17.3 \pm 2.6 vs $16.3 \pm 2.1)$ and cristae density ( $21.6 \pm 2$ vs. $23.3 \pm 1.9)$ remained the same. At 60 days of age, the number of mitochondria (18.1 \pm 7.6 vs $1.8 \pm 0.5 ; P<0.01)$ and the cristae density $(27.7 \pm 1.9$ vs. $5.8 \pm 1.2 ; \mathrm{P}<0.01)$ decreased significantly while the size did not change $(7.3 \% \pm 0.2$ vs $8.2 \% \pm 2.5)$. At the early stages of Chkb muscular dystrophy, there is an increase in mitochondrial size but not number or morphology. As the disease progresses, the increase in mitochondrial size remains, however the number of mitochondria, and the cristae within mitochondria, decrease (Supplementary Fig. 3a, b). 


\section{Chkb deficiency results in increased lipid droplet accumulation in differentiated myocytes in culture}

To address if the observed increase in TG in $\mathrm{Chkb}^{-/}$mice was due to muscle specific events or was due to larger physiological changes that then impact muscle physiology, we assessed TG level in primary cultured muscle cells subsequent to myoblast differentiation.

We first determined if Chkb deficiency alters differentiation in primary myoblasts. Primary muscle cell cultures were examined for their transition from a single cell proliferative condition to differentiated multinucleated myotubes. During the process of differentiation, mononuclear myoblasts fuse to form myocytes (myotubes), which are large multinucleated cells. We isolated skeletal myoblasts from $\mathrm{Chkb}^{+/+}$and $\mathrm{Chkb}{ }^{-/}$mice and induced differentiation by switching to low growth factor serum. Representative light micrographs of cultures of dissociated myogenic cells from skeletal muscle of $\mathrm{Chkb}^{+/+}$ and $\mathrm{Chkb}^{-/}$mice at 0,3 and 5 days after switching to differentiation media show a similar degree of myotube formation (Fig. 6a). Chkb deficiency resulted in a compensatory upregulation of Chka gene expression as well as a significant increase in the markers of myocyte injury, namely Icam 1 and $\operatorname{Tgfb} 1^{26}$ (Fig. 6b). We calculated the fusion index, which is nuclei distribution, to determine the extent of myotube differentiation, by immunofluorescence staining. There was no difference between the $\mathrm{Chkb}^{+/+}$and $\mathrm{Chkb}^{-/}$cells in terms of the percentage of nuclei within the myotubes, the average number of nuclei in each myotube, or the distribution of nuclei in myotubes 
(Fig. c, d). Loss of Chkb function does not appear to affect gross myoblast differentiation.

To assess whether Chkb deficiency modulates TG storage in isolated myotubes, we stained differentiated myotubes with BODIPY 493/503 to visualize neutral lipid droplets. Lipid droplets were noticeably more abundant and larger in Chkb deficient myotubes compared to wild type (Fig. 6e). Quantification of the corrected total cell fluorescence intensity in $\mathrm{Chkb}^{-/}$myotubes confirmed a significant 2-fold increase in lipid droplet formation (Fig. 6f). The increase in TG level in differentiated muscle cells isolated from $\mathrm{Chkb}^{-/}$mice is in line with the increased TG and lipid droplet levels observed in isolated hindlimb muscle from older $\mathrm{Chkb}^{-/}$mice, and implies that the increase in TG in hindlimb muscle due to the loss of Chkb function is a direct effect on lipid metabolism within the muscle cells themselves.

\section{Changes in expression of peroxisome proliferator-activated receptors (Ppars) and target genes reinforce the observed changes in lipid levels in Chkb ${ }^{-/}$hindlimb muscle}

Our lipidomic data imply a temporal shift from the use of fatty acids for energy to lipid storage in affected muscle of $\mathrm{Chkb}^{-/}$mice. To further investigate this metabolic shift, we determined the expression of peroxisome proliferator-activated receptors (Ppars) in affected muscle of 30 day old $\mathrm{Chkb}^{+/+}, \mathrm{Chkb}^{+/}$and $\mathrm{Chkb}^{-/-}$mice. Peroxisome proliferatoractivated receptors (PPARs) are master regulators of lipid metabolism ${ }^{27,28}$. The endogenous ligands for Ppars are fatty acids and their derivatives. There are three Ppar members, each encoded by distinct genes, designated Ppara, Pparb/d and Pparg. 
Ppara and Pparb/d primarily regulate the expression of genes required for fatty acid oxidation, with Pparb/d also regulating genes required for mitochondria biogenesis. Pparg is primarily expressed in adipose tissue and regulates insulin sensitivity and glucose metabolism ${ }^{27}$. Using reverse transcription (RT) qPCR, we determined that the expression of Ppara and Pparb/d were 4-fold and 6-fold lower, while Pparg was 2-fold higher, in the hindlimb muscle of 30 day old mice $\mathrm{Chkb}^{-/}$mice compared to wild type (Fig. 7a). Consistent with RT qPCR results, assessment of Ppar protein levels by western blot show decreased Ppara and Pparb/d protein expression, and an increase in Pparg protein expression, in Chkb deficient hindlimb muscle compared to wild type (Fig. 7b). Ppar protein levels did not change in Chkb deficient forelimb muscle compared to $\mathrm{Chkb}^{+/+}$(Supplementary Fig. 4) indicating that the Ppar changes are isolated to affected muscle.

To further evaluate and validate how the Ppar pathway contributes to the lipid metabolic changes observed in $\mathrm{Chkb}^{-/}$mice, we utilized a microarray of $82 \mathrm{Ppar}$ regulated genes, along with 4 housekeeping genes, to assess transcriptional changes in the hindlimb muscle of 30 day old mice. As expected, there was no change in the expression of the 4 housekeeping genes. For Ppar receptors to bind to Ppar response elements in gene promoters, Ppars form obligate heterodimers with Retinoid $\mathrm{X}$ receptors (Rxr). There are three members of the Rxr family, Rxra, Rxrb, and Rxrg, and their expression was reduced 8-, 5-, and 16-fold in hindlimb muscle of $\mathrm{Chkb}^{-/-}$mice compared to wild type (Fig. 7c, d). Ppar and Rxr heterodimers are bound to DNA with coactivator molecules ${ }^{29}$ and the expression of each co-activator was also decreased from 2.8- to 14.4-fold compared to wild type (Fig. 7c, d). The several fold decrease in 
expression of the Ppars, as well as their obligate co-receptors, aligns well with the observed changes in lipid profiles we observed in affected muscle of $\mathrm{Chkb}^{-/-}$mice that predict a decreased capacity to import and use fatty acids by mitochondria for $\beta$ oxidation.

Among Ppar associated genes, the expression of 44 genes was decreased statistically significantly $(P<0.05)$ by at least 2 -fold in $\mathrm{Chkb}^{-/}$mice, while 8 genes were upregulated at least 2-fold (Fig. 7 c-f, Supplementary Fig. 5, Supplementary Table1 and Table2). Carnitine palmitoyltransferase $1 \mathrm{~b}$ (Cpt1b), the major muscle isoform of Cpt, is involved in the carnitine shuttle as it catalyzes the conversion of cytoplasmic long-chain fatty acyl-CoA and carnitine into AcCa that are translocated across the inner mitochondrial membrane for subsequent mitochondrial fatty acid $\beta$-oxidation. The expression of Cpt1b was decreased 7.9-fold in affected muscle of $\mathrm{Chkb}^{-/}$mice. In addition, the expression of enzymes required for mitochondrial fatty acid $\beta$-oxidation were also decreased several fold in affected muscle of $\mathrm{Chkb}^{-/}$mice including several fatty acylCoA synthases/ligases, fatty acid binding proteins, and fatty acid $\beta$-oxidation enzymes.

Ppara and Ppar b/d are the major transcriptional reporters that regulate expression of fatty acid metabolizing genes. The many-fold decrease in the expression of these Ppars that was specific to affected muscle, along with their coreceptors and downstream target genes corroborate the lipdomics data that suggest that the major change in lipid metabolism in Chkb mediated muscular dystrophy is an inability to metabolize fatty acids via mitochondrial $\beta$-oxidation resulting in shunting of excess fatty acid into TG rich lipid droplets. 


\section{Discussion}

Lipid metabolism is highly integrated. Fluctuating levels of lipid metabolites can not only alter shunting of lipids between tangential pathways, but lipids can also directly bind to transcription factors and alter gene expression of lipid metabolic genes. This study highlights these metabolic factors by determining that inactivation of a gene for PC synthesis does not alter PC level. Indeed, the changes in the level of PC do not appear to contribute to the disease phenotype. This study proposes (1) that a change in PC level is not the major metabolic driver behind this disease despite the fact that the genetic defect lies within the major metabolic pathway for the synthesis of PC, (2) a mechanistic model for defective muscle lipid metabolism in $\mathrm{Chkb}^{-/-}$mice in which the balance between storage and usage of fatty acids is disrupted, and (3) a mechanism for the rostral-to-caudal gradient for Chkb mediated muscular dystrophy.

Importantly, we report that at an early stage of Chkb mediated muscular dystrophy, there is a 12 - to 15 -fold increase in the levels of the mitochondrial specific lipids CL and AcCa. Importantly, these changes were observed only in affected muscle of $\mathrm{Chkb}^{-/}$mice. As the disease progresses, $\mathrm{AcCa}$ and $\mathrm{CL}$ levels return to wild type, and a 12-fold increase in the storage lipid TG occurs. The increase in the mitochondrial specific phospholipid $C L$ is quite telling as far as disease progression. Our TEM of mitochondria in affected muscle during the early stage of Chkb mediated muscular dystrophy revealed a similar number of mitochondria with intact cristae in compared to wild type, however, there was a substantive increase in large mitochondria in affected muscle of $\mathrm{Chkb}^{-/}$mice. We propose that the large increase in $\mathrm{CL}$ in affected muscle of 
$\mathrm{Chkb}^{-/}$mice in the early stage of the disease are mainly driven by the increase in mitochondrial size. As the mice aged the level of CL decreased and had returned to that of wild type by 30 days. At 30 days, mitochondrial size was still increased, however, the number of mitochondria, as well as their cristate (where the bulk of CL resides) were substantively decreased, providing a reasonable explanation for $C L$ mass being

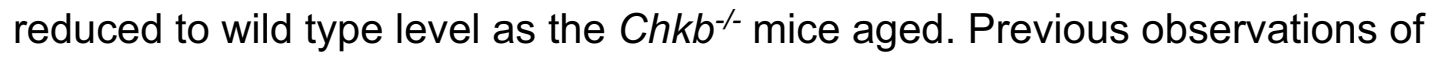
mitochondria in $\mathrm{Chkb}^{-/}$mice have only been determined in mice with advanced disease $e^{6,30}$, where similar changes in mitochondrial morphological features and numbers were observed. Indeed, one would predict that as $\mathrm{Chkb}^{-/}$mice aged there would be a further decrease in CL mass as mitochondrial numbers further decrease.

Beyond the large increase in CL mass, the other major change in lipid level at the early stage of Chkb mediated muscular dystrophy was a 15-fold increase in AcCa level in affected muscle. This implies that there is either a decreased ability to transport of AcCa into mitochondria for subsequent fatty acid $\beta$-oxidation, and/or incomplete $\beta$ oxidation resulting in a backup of substrate within this pathway. In support of this idea the expression of many of the enzymes required for fatty acid transport into mitochondria and subsequent fatty acid $\beta$-oxidation were decreased many fold in affected muscle of $\mathrm{Chkb}^{-/}$mice. The increase in AcCa level at the early stage of Chkb mediated muscular dystrophy, and the decreased expression of genes required for its synthesis and use, is consistent with an inability to import AcCa into mitochondria for fatty acid $\beta$-oxidation.

As $\mathrm{Chkb}^{-/}$mice aged, $\mathrm{AcCa}$ and $\mathrm{CL}$ and levels in hindlimb muscle returned to wild type and by 30 days a dramatic 12 -fold increase in TG level was observed. The 
increase in TG level is consistent with impaired AcCa uptake into mitochondria resulting in a shunting of fatty acids from energy source to energy storage ${ }^{31}$. This observation is consistent with other reports showing that inhibition of PC biosynthesis in mouse liver, and cell culture, significantly increased TG level ${ }^{32,33}$. One interesting additional observation from our study was that $\sim 80 \%$ of the photographed lipid droplets from Chkb-/ hindlimb muscles were closely associated with mitochondria (Fig. 5a and Supplementary Fig. 2c). A recent study by Benador et al. demonstrated that peri-droplet mitochondria have enhanced bioenergetic capacity and reduced fatty acid oxidation capacity, and that peri-droplet mitochondria promote lipid droplet expansion by providing ATP for triglyceride synthesis ${ }^{34}$. Our observation of an increase in peri-droplet mitochondria in $\mathrm{Chkb}^{-/}$affected skeletal is consistent with this study, and with our proposal that there is a reprogramming of muscle lipid metabolism from impaired fatty acid usage for energy to fatty acid storage in TG.

Two perplexing aspects of Chkb mediated muscular dystrophy are (i) PC level does not change despite the fact that the sole genetic defect is inactivation of a gene that encodes the first step of the PC biosynthetic pathway, and (ii) the rostral-to-caudal gradient of the disease. We propose that the rostral-to-caudal gradient of the disease is due to compensatory effects modulated by a second choline kinase isoform, Chka. In hindlimb muscle of $\mathrm{Chkb}^{-/}$mice we observed a marked reduction in Chka protein level, while conversely in the forelimb of $\mathrm{Chkb}^{-/}$mice there was a compensatory upregulation of Chka. The ability of an increase in Chka level to protect against Chkb-mediated muscular dystrophy is consistent with previous work that demonstrated that the viral delivery of the Chka gene improves dystrophic phenotypes in Chkb-/ mice with 
comparable potency to rescue by delivery of $C h k b{ }^{35}$. One caveat to the above is the fact that PC level was not different between the forelimb and hindlimb muscle of $C h k b^{-/-}$ mice, nor was it different between wild type and $\mathrm{Chkb}^{-/-}$mice at any stage of the disease. This suggests that PC supply must be able to be replenished at a step downstream of choline kinase. We further propose that PC level does not change as PC can be replenished via exogenous PC supply. PC is imported into cells from serum via low density lipoproteins (LDL), and enhanced expression of scavenger receptor-B1 (SRB1) and low-density lipoprotein receptor (LDLR) was previously observed in muscle of $C h k b^{-/}$mice, both of which would be expected to enhance the uptake of plasma PC ${ }^{7}$. We propose that the expected decrease in the level of PC in hindlimb muscle of $\mathrm{Chkb}^{-/}$ mice, due to inactivation of the Ckkb gene and downregulation of Chka gene expression, is not observed as this can be compensated for by increased PC uptake from serum. These predictions are consistent with the changes in muscle function along the rostral-to-caudal gradient in $\mathrm{Chkb}^{-/}$mice.

A interesting mechanistic aspect is the transition from an inability to synthesize $\mathrm{PC}$ in affected muscle to an increase in AcCa. The synthesis of $\mathrm{PC}$ requires the consumption of DAG at the final step in the CDP-choline pathway, and this would not occur in affected muscle as the choline kinase step is either inactivated (Ckhb) or downregulated (Chka). DAG requires fatty acids for its synthesis, and an inability to synthesize DAG could result in an inability to utilize fatty acids for subsequent DAG synthesis. Indeed, over time we see an increase in DAG mass in affected muscle. The inability to synthesize PC results in a metabolic defect downstream within this pathway that results in major changes in tangential yet connected lipid metabolic pathways over 
time, an inability to use excess fatty acid for energy followed by its storage as neutral lipid.

\section{Acknowledgments}

We acknowledge funding support from the Canadian Institutes for Health Research (to CRM) and the Atlantic Innovation Fund (to CRM and EH). We thank Gregory Cox for sharing Chkb mice.

\section{AUTHOR CONTRIBUTIONS}

MT, JPFM, KN, EPH, and CRM conceived the study. MT, SL, SS, KU, JR, SS, MP, AM, MSM, AAM and MM performed experiments. MT, JPFM, and CRM wrote the paper.

\section{DECLARATION OF INTERESTS}

The authors declare no competing interests. 
FIGURE LEGENDS

Fig. 1 | Glycerophospholipid pathway, maintaining the balance between storage and usage of fatty acids. Fatty acids derived from the plasma are first activated by esterification to fatty acyl-CoA. Subsequently, they will either used as energy through mitochondrial beta oxidation or funneled into phosphatidic acid (PA) synthesis. PA is the simplest glycerophospholipid and contains only a phosphate group as a hydrophilic moiety. A pool of PA can either 1. enter CDP-DAG pathway to generate phosphatidylinositol $(\mathrm{PI})$, phosphatidylglycerol $(\mathrm{PG})$ and cardiolipin $(\mathrm{CL})$ or 2. be converted to diacylglycerol (DG). In fact, the majority of the de novo synthesized PA is dephosphorylated into DG. DG can then be used to catalyze the production of triacylglycerides (TG) and stored as energy source in lipid droplets or be converted into the two most abundant membrane phospholipids: phosphatidylcholine (PC) and phosphatidylethanolamine (PE) as well as phosphatidylserine (PS). PC and PE are generated when CDP-choline or CDP-ethanolamine are combined with DG (not shown). The CDP-choline pathway (Kennedy pathway) begins with the uptake of exogenous choline into the cell. The first enzymatic reaction is catalyzed by choline kinases (Chka and Chkb) and involves the phosphorylation of choline to form phosphocholine.

Fig. 2 | Choline kinase deficient mice display hallmark muscular dystrophy Phenotypes. a, Body weight was recorded each week at similar times over the entire duration of phenotyping experiment for $\mathrm{Chkb}^{+/+}, \mathrm{Chkb}^{+/}$and $\mathrm{Chkb}^{-/}$mice. b, Grip 
strength measurements were performed at 3 different timepoints and normalized to body weight (BW). c, Total distance run during an exhaustion test for all experimental groups at 3 different timepoints. d, Serum creatine kinase (CK) level measurements of 15-week-old $\mathrm{Chkb}^{+/}, \mathrm{Chkb}^{+/}$and $\mathrm{Chkb}^{-/}$mice. e, Loss in muscle force as a result of repeated contractions of EDL muscles by direct stimulation of the nerve for each

genotype. f, Maximal specific force generated by freshly isolated extensor digitorum longus (EDL) muscle for each genotype All values are expressed as means \pm SEM; $n=6-13$ animals per group. Significance was calculated using one-way ANOVA with Tukey's multiple comparison test for each specific time point. ${ }^{*} P<0.01$ vs. all the other groups and \#P<0.05 vs. Chkb ${ }^{+/+}$group at each specific timepoint.

\section{Fig. 3 | Chka protein expression is inversely correlated with the rostro-caudal} gradient of severity in Chkb-mediated muscular dystrophy

Western blot of $\mathbf{a}$, forelimb (triceps) and $\mathbf{b}$, hindlimb (quadriceps) samples from three distinct (lanes 1-3) $\mathrm{Chkb}^{+/+}$, four distinct (lanes 4-7) $\mathrm{Chkb}^{+/}$and three distinct (lanes 810) $\mathrm{Chkb}^{-/}$mice probed with anti-Chka, anti-Chkb, and anti-Gapdh antibodies. Bottom: densitometry of the WB data shows the ratio of Chka and Chkb to Gapdh. Chka signal is not significantly different in forelimb and hindlimb samples from $\mathrm{Chkb}^{+/}$mice compared to the wild type. Chka is upregulated in forelimb muscles and downregulated in hindlimb muscles from Chkb-/ mice. Chkb signal is decreased in hindlimb and forelimb muscle samples of $\mathrm{Chkb}^{+/-}$mice and is absent in muscle samples of $\mathrm{Chkb}^{-/-}$ mice. Values are means $\pm S D ; n=3-4$ per group. ${ }^{*} P<0.01$ vs $C h k b^{+/+},{ }^{* *} \mathrm{P}<0.01$ vs all the 
other groups (one-way ANOVA with Tukey's multiple comparison test). \#P<0.05 vs $\mathrm{Chkb}^{+/+}$(Student's t-test).

Fig. 4 | Loss of Chkb activity exerts a major effect on neutral lipid abundance. Comparison of expression levels of major glycerophospholipids and AcCa between the $\mathrm{Chkb}^{+/+}$and $\mathrm{Chkb}^{-/-}$mice. The analysis was performed on $\mathbf{a - b}, 12$ days old forelimb (triceps), c-d, 12 days old hindlimb (quadriceps) and e-f, 30 days old hindlimb (quadriceps) samples. $\mathbf{b}, \mathbf{d}$ and $\mathbf{f}$. Summary of fold change and statistical tests performed on major glycerophospholipids. $n=3$ mice per group. Pairwise Wilcoxon signed rank test with Bonferroni correction was used to determine the significance of a median pair-wise fold-increase in lipid amounts at an overall significance level of $5 \%$. As the Bonferroni correction is fairly conservative, significant differences are reported at both pre-correction $\left({ }^{*}\right)$ and post-correction $\left({ }^{* * *}\right)$ significance levels. AcCa, acylcarnitine; TG, triacylglycerol; DG, diacylglycerol; PC, phosphatidylcholine; PE, phosphatidylethanolamine; PG, phosphatidylglycerol; PI, phosphatidylinositol; PS, phosphatidylserine.

Fig. 5 | Increased intramyocellular lipid droplet accumulation and enlarged mitochondria in hindlimb muscles from $\mathrm{Chkb}^{-/-}$mice. a, Transmission electron microscopy (TEM) appearance of the hindlimb muscle samples (quadriceps) of $C h k b^{+/+}$ and $\mathrm{Chkb}^{-/}$mice at 12 days and 115 days of age. (representative of 3 mice per group). LD=Lipid droplets. M=Mitochondria. ${ }^{*}=$ Disrupted sarcomeres. b, Quadriceps muscle sections of 30 days old $\mathrm{Chkb}^{+/+}$and $\mathrm{Chkb}^{-/}$mice were fixed and stained with BODIPY- 
493/503 to visualize LDs (Green). Concanavalin A dye conjugate (CF ${ }^{\mathrm{TM}}$ 633) and DAPI were used to stain membrane (Red) and nucleus (Blue) respectively. (representative of 3 mice per group).

Fig. 6 | Chkb deficiency results in increased lipid droplet accumulation in differentiated myocytes in culture. a, Representative image of isolated skeletal myoblasts from $\mathrm{Chkb}^{+/+}$and $\mathrm{Chkb}^{-/-}$mice, cultured on Matrigel $\AA^{\circledR}$ coated culture flasks. At day 0 , when the cells reached $80 \%$ confluency, the medium was replaced by differentiation medium and maintained in differentiation media for 5 days. b, RT-qPCR analysis of gene expression in isolated myocytes from $\mathrm{Chkb}^{+/+}$and $\mathrm{Chkb}^{-/}$mice at day 5 of differentiation. Values are means $\pm S D ; n=3$ independent experiments. ${ }^{*} P<0.05,{ }^{* *} P<$ 0.01. Student's t-test. Formaldehyde fixed and immunostained myotubes were categorized into three groups ( 1 to 3 nuclei, 4 to 10 nuclei, and $>10$ nuclei per myotube). The distribution of nuclei (c), and number of multinuclear myotubes in the two groups (d) were calculated. e, Isolated primary myocytes from $\mathrm{Chkb}^{+/+}$and $\mathrm{Chkb}^{-/-}$mice were fixed 5 days after differentiation and stained with BODIPY-493/503 to visualize LDs (Green). DAPI was used to stain nucleus (Blue). f, The corrected total cell fluorescence intensity of lipid droplets was significantly enhanced in $\mathrm{Chkb}^{-/-}$myotubes. For $\mathbf{c}, \mathbf{d}$ and $\mathbf{f}$, total 100 cells were quantified per group in 3 independent experiments. Data are mean $\pm \mathrm{SD} .{ }^{*} \mathrm{p}<0.05$. Student's t-test.

Fig. 7 | Chkb regulates the gene expression of the members of the Ppar family as well as Ppar target genes. a. Relative gene expression of the Ppar family members. 
b. Western blot of hindlimb (quadriceps) samples from three distinct (lanes 1-3) $\mathrm{Chkb}^{+/+}$, four distinct (lanes 4-7) $\mathrm{Chkb}^{+/}$and three distinct (lanes 8-10) Chkb-/ mice probed with anti-Ppara, anti- Pparb, anti-Pparg, anti-Cpt1b and anti-Gapdh antibodies. Bottom: densitometry of the western blot data shows the ratio of Ppara, Pparb, Pparg and Cpt1b to Gapdh. Values are means \pm SD; $n=3-4$ per group. ${ }^{*} \mathrm{P}<0.01$ vs $C h k b^{+/+}$, ${ }^{* *} \mathrm{P}<0.01$ vs all the other groups (one-way ANOVA with Tukey's multiple comparison test). c, Fold-Change $\left(2^{\wedge}(-\right.$ Delta Delta CT $\left.)\right)$ is the normalized gene expression $\left(2^{\wedge}(-\right.$ Delta CT)) in the Chkb deficient hindlimb sample divided the normalized gene expression $\left(2^{\wedge}(-\right.$ Delta $\left.C T)\right)$ in the control sample. Fold-change values greater than one indicates a positive- or an up-regulation. Fold-change values less than one indicate a negative or down-regulation, and the fold-regulation is the negative inverse of the foldchange. The $p$ values are calculated based on a Student's t-test of the replicate $2^{\wedge}(-$ Delta CT) values for each gene in the $\mathrm{Chkb}^{+/+}$group and $C h k b^{-/}$groups. ${ }^{*} \mathrm{p}<0.05$, ${ }^{* *} \mathrm{p}<0.01$. N=3 samples per group. $\mathbf{d}$, The clustergram of the Ppar family, $R x r$ family and Ppar coactivators across three genotypes. e, Fold change, normalized gene expression for the genes involved in peroxisomal and mitochondrial beta oxidation in the Chkb deficient hindlimb sample divided the normalized gene expression in the control sample. f, The clustergram of the Ppar family, Rxr family and Ppar coactivators across three genotypes. Average arithmetic mean of the expression of 4 housekeeping genes (Actb, B2m, Gusb and Hsp90ab1) were used to normalize the expression of all the studied genes. 


\section{Methods}

Mouse strains.

All animal procedures were approved by the Dalhousie University's Committee on Laboratory Animals in accordance with guidelines of the Canadian Council on Animal Care Guide to the Care and Use of Experimental Animals (CCAC, Ottawa, ON, Canada: vol. 1, 2nd ed., 1993; vol. 2, 1984. Chkb mutant mice in C57BL/6J background were a kind gift of Professor Gregory A. Cox and were originally generated at the Jackson Laboratory (Bar Harbor, Maine, USA) ${ }^{5}$. Male $\mathrm{Chkb}^{+/}$mice on the C57BL/6J background were crossed with female $\mathrm{Chkb}^{+-}$on the same background to generate $\mathrm{Chkb^{+/+ }}$, Chkb ${ }^{-/}$ and $\mathrm{Chkb}^{+/-}$littermates. The mutation identified in $\mathrm{Chkb}^{-/}$mice is a $1.6 \mathrm{~kb}$ genomic deletion between exon 3 and intron 9 that results in expression of a truncated mRNA and the absence of Chkb protein expression ${ }^{5}$.

\section{Mouse genotyping.}

The mutation identified in $\mathrm{Chkb}^{-/-}$mice is a $1.6 \mathrm{~kb}$ genomic deletion between exon 3 and intron $9^{5}$. AccuStart ${ }^{\mathrm{TM}}$ II Mouse Genotyping Kit (Beverly, MA, USA) was used to extract DNA from ear punches and to perform PCR analysis. A single genotyping program was used to amplify both the wild type Chkb allele between exons 5 and 9 and the truncated Chkb allele between exons 2 and 10. The primers used for genotyping were purchased from Integrated DNA Technologies (Coralville, IA, USA). The primer sequences to genotype wild type are Forward Primer: 5'-GTG GGT GGC ACT GGC 
ATT TAT -3'; Reverse Primer: 5'-GTT TCT TCT GTT CCT CTT CGG AGA-3' (amplicon size $753 \mathrm{bp})$.

The primer sequences to genotype the mutants are: Forward Primer: 5'-TAC CCA CGT ACC TCT GGC TTT T -3' Reverse Primer: 5'-GCT TTC CTG GAG GAC GTG AC 3'(amplicon size $486 \mathrm{bp}$ ). For each mouse, one PCR reactions was performed using both the primer sets. If two bands were observed, the mouse was characterized as a heterozygous.

\section{In vivo grip strength and fatigability measurements.}

Forelimb grip strength was measured using a grip strength meter (Columbus Instruments, Columbus, $\mathrm{OH}, \mathrm{USA})$ at 3 time points $(6,12,18$ weeks old) as previously described ${ }^{40}$. All mice were acclimated for a period of five consecutive days before testing. For each time point, Force measurements were collected in the morning hours over a 5-day period, with maximum values for each day over this period averaged to obtain absolute GSM values (Kgf) or normalized to BW (recorded on the first day of testing) for normalized GSM values (Kgf/kg). For the treadmill exhaustion assay, mice are subjected to an enforced running paradigm that tests the resistance level of fatigue in mice. The exhaustion test was performed at 3 time points $(7,13,19$ weeks old) in each group. Groups of mice were made to run on a horizontal treadmill for 5 min at 5 $\mathrm{m} / \mathrm{min}$, followed by an increase in the speed of $1 \mathrm{~m} / \mathrm{min}$ each minute. The total distance run by each mouse until exhaustion was measured. Exhaustion was defined as the inability of the mouse to continue running on the treadmill for 30 seconds, despite repeated gentle stimulation. 


\section{Primary myoblast isolation, culture and differentiation.}

We followed a protocol outlined in Shahini et al. ${ }^{41}$ for isolation of myoblast by enabling the outgrowth of these cells from muscle tissue fragments of $\mathrm{Chkb}^{+/+}$and $\mathrm{Chkb}^{-/}$mice. Briefly, the mice were euthanized via $\mathrm{CO} 2$, were sprayed with $70 \%$ ethanol and transferred to a sterile hood. The forelimb and hindlimb muscles were removed, finely minced into small pieces and transferred to a $50 \mathrm{ml}$ conical tube. $1 \mathrm{ml}$ enzymatic solution of PBS containing collagenase type II $(500 \mathrm{U} / \mathrm{mL})$, collagenase $\mathrm{D}(1.5 \mathrm{U} / \mathrm{mL})$, dispase II $(2.5 \mathrm{U} / \mathrm{mL})$, and $\mathrm{CaCl}_{2}(2.5 \mathrm{mM})$ was added to the tube. The muscle mixture was placed in a water bath at $37^{\circ} \mathrm{C}$ for 60 minutes with agitation every 5 minutes. The suspension was centrifuged for 10 minutes at $300 \mathrm{~g}$. Following centrifugation, the supernatant was removed and discarded, and the pellet was resuspended in proliferation medium. Proliferation medium composed of high glucose Dulbecco's Modified Eagle Medium (DMEM, Gibco, Grand Island, NY), 20\% fetal bovine serum (FBS, Atlanta Biologicals, Flowery Branch, GA), 10\% horse serum (HS, Gibco), 0.5\% chicken embryo extract (CEE, Accurate Chemical and Scientific, Westbury, NY), 2.5 ng/mL bFGF (ORF Genetics, Iceland), $10 \mu \mathrm{g} / \mathrm{mL}$ gentamycin (Gibco), and 1\% AntibioticAntimitotic (AA, Gibco), and $2.5 \mu \mathrm{g} / \mathrm{mL}$ plasmocin prophylactic (Invitrogen, San Diego, CA). The re-suspended pellet containing small pieces of muscle tissue was plated on matrigel coated flasks at $10-20 \%$ surface coverage and incubated at $37^{\circ} \mathrm{C}$ and $5 \% \mathrm{CO}_{2}$ to allow attachment of the tissues to the surface and subsequent outgrowth and migration of cells. The myogenic cell population was further purified with one round of pre-plating on collagen coated dishes to isolate fibroblasts from myoblasts. To induce 
differentiation into multinucleated myotubes, the cells were seeded at $10000 \mathrm{cells} / \mathrm{cm}^{2}$ on plastic coverslip chambers coated with Matrigel and the medium was replaced by differentiation medium containing DMEM with high glucose and $5 \% \mathrm{HS}$.

\section{Ex vivo force measurement.}

At the end of the in vivo phase (Week 19), mice were deeply anesthetized with ketamine and xylazine (80 and $10 \mathrm{mg} / \mathrm{kg}$ ). The extensor digitorum longus (EDL) muscle of the right hindlimb was removed for comparison of Ex vivo force contractions between groups as previously described ${ }^{42,43}$. Briefly, the EDL muscle was securely tied with braided surgical silk at both tendon insertions to the lever arm of a servomotor/force transducer (model 305B) (Aurora Scientific, Aurora, Ontario, Canada) and the proximal tendon was fixed to a stationary post in a bath containing buffered Ringer solution (composition in mM: $137 \mathrm{NaCl}, 24 \mathrm{NaHCO}_{3}, 11$ glucose, $5 \mathrm{KCl}, 2 \mathrm{CaCl}_{2}, 1 \mathrm{MgSO}_{4}, 1$ $\mathrm{NaH}_{2} \mathrm{PO}_{4}$ and 0.025 turbocurarine chloride) maintained at $25^{\circ} \mathrm{C}$ and bubbled with $95 \%$ $\mathrm{O}_{2}-5 \% \mathrm{CO}_{2}$ to stabilize $\mathrm{pH}$ at 7.4. At optimal muscle length, the maximal force developed was measured during trains of stimulation (300 milliseconds, ms) with increasing frequencies up to $250 \mathrm{~Hz}$ or until the highest plateau was achieved. The force generated to obtain the highest plateau was used to determine specific force (maximal force normalized to cross-sectional area of the muscle). Finally, the muscle was subjected to a fatigue protocol consisting of 60 isometric contractions for $300 \mathrm{~ms}$ each, once every 5 seconds. The frequency at which the EDL muscles were stimulated is $250 \mathrm{~Hz}$. The force was recorded every 10th contraction during the repetitive contractions and again at 5 and 10 min afterward to measure recovery. 


\section{Creatine kinase (CK) serum levels.}

CK was determined from serum taken from blood samples withdrawn by cheek bleed at 3 time points (5, 10 and 15 weeks old). Blood was centrifuged for $3000 \mathrm{~g}$ for $10 \mathrm{~min}$ at $4^{\circ} \mathrm{C}$ to obtain the serum. CK determination was performed by standard spectrophotometric analysis, using a CK diagnostic kit (Cat. no. C7522-450, PONITSCIENTIFIC, Canton, MI, USA) according to the manufacturer instructions.

Total RNA isolation, cDNA generation, and quantitative real-time RT qPCR. Isolated tissue samples were incubated overnight in pre-chilled RNAlater® (Cat. no. R0901, Sigma-Aldrich, Ontario, Canada) at $4^{\circ} \mathrm{C}$. Tissues were then homogenized in TRIzol reagent (Cat. no. 15596026, Invitrogen, MA, USA) and total RNA was isolated according to the manufacturer's protocol. Nine hundred nanograms of total RNA was reverse transcribed using High-Capacity cDNA Reverse Transcription Kit@ (Cat. no. 4368814, Applied Biosystems, MA, USA). Quantitative real-time RT-PCR assays were performed on the Bio-Rad CFX96 Touch Real-Time PCR Detection (Bio-Rad ®, California, USA) System using TaqMan ${ }^{\mathrm{TM}}$ Fast Advanced Master Mix (Cat. no. 4444557) and TaqMan $^{\text {TM }}$ Gene Expression Assays (Cat. no. 4331182, ThermoFisher Scientific) for Chka (RRID: Mm00442759_m1), Chkb Cpt1b (Exon boundary7-8)(RRID: Mm01308102_g1), Cnsk2a2 (RRID: Mm01243455_m1), Cpt1b (RRID:

Mm00487191_g1), Gapdh (RRID: Mm99999915_g1), Icam1 (RRID: Mm00516023_m1), Ppara (RRID: Mm00440939_m1), Ppard (RRID: Mm00440940_m1), Pparg (RRID: 
Mm00440940_m1) and Tgfb1 (RRID: Mm011778820_m1). Reactions were run in triplicate.

\section{Microarray analysis of Ppar targets}

Mature RNA was isolated using Qiagen RNeasy Plus Mini Kit (Cat. no 74134) according to the manufacturer's instructions. RNA quality was determined using a spectrophotometer and was reverse transcribed using a cDNA conversion kit. The cDNA was used on the real-time RT2 Profiler PCR Array (QIAGEN, Cat. no. PAMM149Z) in combination with RT2 SYBR® Green qPCR Mastermix (Cat. no. 330529). CT values were exported to an Excel file to create a table of CT values. This table was then uploaded on to the data analysis web portal at http://www.qiagen.com/geneglobe. Samples were assigned to controls and test groups. CT values were normalized based on a/an Manual Selection of reference genes. The data analysis web portal calculates fold change/regulation using delta delta CT method, in which delta CT is calculated between gene of interest ( $\mathrm{GOI}$ ) and an average of reference genes (HKG), followed by delta-delta CT calculations (delta CT (Test Group)-delta CT (Control Group)). Fold Change is then calculated using $2^{\wedge}$ (-delta delta CT) formula. The data analysis web portal to plot scatter clustergram, and heat map.

\section{Lipid extraction}

We performed lipid extractions using the modified Bligh and Dyer extraction for LC-MS analysis of lipids protocol ${ }^{44}$. All reagents were of LC-MS grade. Briefly, the muscle tissue $(\sim 10 \mathrm{mg})$ was homogenized with a steel bead in $1 \mathrm{ml}$ of cold $0.1 \mathrm{~N} \mathrm{HCl}$ :methanol 
$(1: 1, v / v)$ using a TissueLyser II instrument (Qiagen) set at 30 strokes/s for $2-4$ min. Based on protein quantification results, all samples were adjusted to the final concentration of $700 \mu \mathrm{g} / \mathrm{ml}$ and spiked with $10 \mu \mathrm{l}$ of internal standard (Avanti Polar Lipids Inc; Catalog Number-330707). $500 \mu \mathrm{l}$ of chloroform was added to each sample, vortexed for 30 minutes and centrifuged to separate phases (5 minutes at $6000 \mathrm{rpm}$ ). The bottom organic phase was transferred into a new Eppendorf and dried under a nitrogen stream. Samples were stored at $-80^{\circ} \mathrm{C}$ until ready for analysis.

\section{UHPLC method for lipid analysis.}

The Accucore C30 column $(250 \times 2.1 \mathrm{~mm}$ I.D., particle size: $2.8 \mu \mathrm{m})$ was obtained from ThermoFisher Scientific (ON, Canada). The mobile phase system consisted of solvent A (acetonitrile: $\mathrm{H} 2 \mathrm{O}$ 60:40 v/v) and solvent B (isopropanol: acetonitrile: water 90:10:1 v/v) both containing $10 \mathrm{mM}$ ammonium formate and $0.1 \%$ formic acid. C30-RPLC separation was carried out at $30^{\circ} \mathrm{C}$ (column oven temperature) with a flow rate of $0.2 \mathrm{~mL} / \mathrm{min}$, and $10 \mu \mathrm{L}$ of the lipid extraction suspended in the mobile phase solvents mixtures $(A: B$, 70:30\%) was injected onto the column. The following system gradient was used for separating the lipid classes and molecular species: $30 \%$ solvent B for 3 min; then solvent $B$ increased to $50 \%$ over $6 \mathrm{~min}$, then to $70 \% \mathrm{~B}$ in 6 min, then kept at $99 \%$ B for 20 min, and finally the column was re-equilibrated to starting conditions ( $30 \%$ solvent A) for 5 min prior to each new injection. 
High resolution tandem mass spectrometry and lipidomics.

Lipid analyses were carried out using a Q-Exactive Orbitrap mass spectrometer controlled by X-Calibur software 4.0 (ThermoScientific, MO, USA) with an acquisition HPLC system. The following parameters were used for the Q-Exactive mass spectrometer - sheath gas: 40 , auxiliary gas: 5 , ion spray voltage: $3.5 \mathrm{kV}$, capillary temperature: $250{ }^{\circ} \mathrm{C}$; mass range: $200-2000 \mathrm{~m} / \mathrm{z}$; full scan mode at a resolution of $70,000 \mathrm{~m} / \mathrm{z}$; top-1 m/z and collision energy of 35 (arbitrary unit); isolation window: $1 \mathrm{~m} / \mathrm{z}$; automatic gain control target: $1 \mathrm{e} 5$. The instrument was externally calibrated to $1 \mathrm{ppm}$ using ESI negative and positive calibration solutions (ThermoScientific, MO, USA). Tune parameters were optimized using a mixture of lipid standards (Avanti Polar Lipids, Alabama, USA) in both negative and positive ion mode Thermo Scientific ${ }^{\mathrm{TM}}$ LipidSearch ${ }^{\mathrm{TM}}$ software version 4.2 was used for lipid identification and quantitation. First, the individual data files were searched for product ion MS/MS spectra of lipid precursor ions. MS/MS fragment ions were predicted for all precursor adduct ions measured within $\pm 5 \mathrm{ppm}$. The product ions that matched the predicted fragment ions within a \pm 5 ppm mass tolerance was used to calculate a match-score, and those candidates providing the highest quality match were determined. Next, the search results from the individual positive or negative ion files from each sample group were aligned within a retention time window $( \pm 0.2 \mathrm{~min})$ and the data were merged for each annotated lipid. 


\section{Data cleanup and statistical analysis of lipids.}

Lipid concentrations extracted from the LipidSearch software were further analyzed with an in-house script using the $\mathrm{R}$ programming language. The data was filtered to exclude any peak concentration estimates with a signal to noise ratio (SNR parameter) of less than 2.0 or a peak quality score (PQ parameter) of less than 0.8 . If this exclusion resulted in the removal of two observation within a biological triplicate, the remaining observation was also excluded. The individual concentrations were then gathered together by lipid identity (summing together the concentration of multiple mass spectrometry adducts where these adducts originated from the same molecular source and averaging together biological replicates) and grouped within the broader categories of AcCa, TG, DG, PC, PE, PG, CL, PI, PS. The result was nine groups containing multiple lipid concentrations corresponding to specific lipid identities, which were then compared between wild type and KO samples using a (paired, non-parametric) Wilcoxon signed-rank test at an overall significance level of $5 \%$ (using the Bonferroni correction to account for the large number of tests performed). As the Bonferroni correction is fairly conservative, significant differences are reported at both precorrection $\left({ }^{*}\right)$ and post-correction $\left({ }^{* * *}\right)$ significance levels.

\section{Nile red 550 / $640 \mathrm{~nm}$, BODIPY 493/503 nm and nuclei staining of muscle tissue.}

Quadriceps and gastrocnemius muscles were embedded in Optimal Cutting Temperature $^{\mathrm{TM}}$ (Sakura Finetek, Torrence, CA), and were frozen in cooled isopentane in liquid nitrogen and stored at $-80^{\circ} \mathrm{C}$. Frozen sections $(7 \mu \mathrm{m}$ thick) were thaw-mounted on SuperFrost Microscope slides (Microm International, Kalamazoo, MI) and air dried. 
Tissue sections were then fixed in 4\% (w/v) paraformaldehyde for 15 minutes and incubated with Concanavalin A CF® Dye Conjugates CF ${ }^{\mathrm{TM}} 633(50-200 \mu \mathrm{g} / \mathrm{mL})$ for 20 minutes followed by incubation with either Nile red solution in PBS $(0.5 \mu \mathrm{g} / \mathrm{mL})$ or BODIPY 493/503 for 15 minutes. The sections were then washed for 5 times with PBS, each time for 15 minutes and mounted using ProLong ${ }^{\text {TM }}$ Gold Antifade Mountant with DAPI (Thermo Scientific ${ }^{\text {TM }}$, Cat. no. P36931) and cured overnight in the dark. Slides were observed under a confocal microscope (Leica TCS SP8 with LIGHTNING) using excitation wavelength 633 for Concanavalin A, 550nm for Nile red, $448 \mathrm{~nm}$ for BODIPY and 405 for DAPI.

\section{BODIPY 493/503 and nuclei staining of primary myocytes.}

Isolated skeletal myoblasts were cultured on Matrige|® coated Glass chamber slides (Thermo Scientific ${ }^{\mathrm{TM}}$, Cat. no. 154534) and differentiated into myocyte. 3 days after differentiation, the cells were washed two times with PBS and fixed in $4 \%(w / v)$ paraformaldehyde for 15 minutes. The cells were washed with PBS for 10 minutes and incubated with BODIPY solution in PBS for 15 minutes, at room temperature on a shaker. The cells were then washed for 3 times with PBS, each time for 15 minutes and mounted using ProLong ${ }^{\mathrm{TM}}$ Gold Antifade Mountant with DAPI (Thermo Scientific ${ }^{\mathrm{TM}}$, Cat. no. P36931) and cured overnight in the dark. Slides were observed under a confocal microscope (Leica TCS SP8 with LIGHTNING) using excitation wavelength $448 \mathrm{~nm}$ for BoODIPY and 405 for DAPI. Images were converted to 8-bit and the total corrected cellular fluorescence for the green channel was measured in random 100 cells per group using FIJI $(\mathrm{NIH})$ software. The total corrected cellular fluorescence $(\mathrm{TCCF})=$ 
integrated density - (area of selected cell $\times$ mean fluorescence of background readings), was calculated and compared between groups ${ }^{45}$.

\section{Transmission electron microscopy.}

For TEM analysis, $\sim 5 \times 5 \mathrm{~mm}$ cubes of quadriceps, gastrocnemius and triceps were with $2.5 \%$ Glutaraldehyde diluted with $0.1 \mathrm{M}$ sodium cacodylate buffer and postfixed with $1 \%$ osmium tetroxide in Millonig's buffer solution for $2 \mathrm{hr}$, dehydrated, and embedded in epon araldite resin. Ultrathin sections were stained with $2 \%$ uranyl acetate for 30 min and lead citrate for 4 min and viewed with a JEOL JEM 1230 Transmission Electron Microscope at $80 \mathrm{kV}$. Images were captured using a Hamamatsu ORCA-HR digital camera. Three mice per genotype for each timepoint were evaluated. The mitochondrial content was determined from the images at $10,000 \times$ magnification using Image $\mathrm{J}$ software and calculated as mitochondria count/field by blinded investigators. Point counting was used to estimate mitochondrial volume density and mitochondrial cristae density based on standard stereological methods ${ }^{46,47}$. Only mitochondria profiles of acceptable quality defined as clear visibility and no or few missing spots of the inner membrane were included. Using ImageJ software, a point grid was digitally layered over the micrographic images at $20,000 x$ or $40,000 x$ magnification for mitochondrial volume density and cristae density calculations respectively. Grid sizes of $85 \mathrm{~nm} \times 85 \mathrm{~nm}$ and $165 \mathrm{~nm} \times 165 \mathrm{~nm}$ were used to estimate mitochondria volume and cristae surface area, respectively. Mitochondria volume density was calculated by dividing the points assigned to mitochondria to the total number of points counted inside the muscle. The mitochondrial cristae surface area per mitochondrial volume (mitochondrial cristae 
density) was estimated by the formula: mitochondrial cristae density $=(4 / \pi) B A$, where BA is the boundary length density estimated by counting intersections on test lines multiplied by $\pi / 2$. In brief, we counted the intersections I(imi) between the inner mitochondrial membrane trace and the test lines and measured the total length of the test line within the mitochondria profile to calculate mitochondrial cristae density $=2$. I(imi)/L(mi).

\section{Western blot analysis (WB) and quantification.}

The muscle tissue $(\sim 100 \mathrm{mg})$ was homogenized with a steel bead in $1 \mathrm{ml}$ of cold RIPA buffer containing $1 \mathrm{X}$ Proteinase Inhibitor Mix (complete ${ }^{\mathrm{TM}}$ Protease Inhibitor Cocktail, Roche, Cat. no.11 697498 001), 1X PhosStop (Roche, Mannheim Germany, Cat. no.04 906845 001) using a TissueLyser II instrument (Qiagen) set at 30 strokes/s for $2-4$ min. Based on protein quantification results, all samples were adjusted to the final concentration of $2 \mathrm{ug} / \mathrm{ul}$ and heat-denatured for $5 \mathrm{~min}$ at $99^{\circ} \mathrm{C}$ in $2 \mathrm{X}$ Laemmli buffer. Proteins were separated by SDS-PAGE and transferred to nitrocellulose membranes. The membranes were incubated in Odyssey blocking solution for $1 \mathrm{~h}$. Total proteins were detected by probing the membranes with appropriate primary antibodies overnight at $4^{\circ} \mathrm{C}$. The following antibodies were used: Chka (1:1000, Abcam Cat\#ab88053), Ppara (1:1000, Abcam, Cat\#Ab24509), Pparb (1:1000, Biorad, Cat\#AHP1272), Cpt1b (1:1000, Proteintech®, Cat\#22170-1-AP), Chk $\beta$ (1:250, Santa Cruz, Cat\#398957), GAPDH (1:1000, Cell signaling, Cat\#398957), Ppary (1:500, Santa Cruz, Cat\# sc-7273). Proteins were visualized with goat anti-rabbit IRDye-800- or -680secondary antibodies (LI-COR Biosciences) or anti-mouse m-IgGk BP-CFL 790 (Santa 
Cruz, Cat. no.sc-516181) using an Odyssey imaging system and band density were evaluated using FIJI (NIH).

\section{Quantification and statistical analysis.}

All experiments were repeated 3 or more times. Data are presented as mean \pm SEM or mean $\pm \mathrm{SD}$, as appropriate. For comparison of two groups the two-tailed Student's t-test was used unless otherwise specified. Comparison of more than two groups was done by one-way ANOVA followed by the Tukey's Multiple Comparison test. $\mathrm{P}$ values $<0.05$ were considered significant.

\section{Data availability}

All data that support the findings of this study are available from the corresponding authors upon request. 


\section{REFERENCES}

1 Ishidate, K. Choline/ethanolamine kinase from mammalian tissues. Biochim Biophys Acta 1348, 70-78, doi:10.1016/s0005-2760(97)00118-5 (1997).

2 Aoyama, C., Liao, H. \& Ishidate, K. Structure and function of choline kinase isoforms in mammalian cells. Prog Lipid Res 43, 266-281, doi:10.1016/j.plipres.2003.12.001 (2004).

3 Wu, G., Aoyama, C., Young, S. G. \& Vance, D. E. Early embryonic lethality caused by disruption of the gene for choline kinase alpha, the first enzyme in phosphatidylcholine biosynthesis. J Biol Chem 283, 1456-1462, doi:10.1074/jbc.M708766200 (2008).

4 Bansal, D. et al. Defective membrane repair in dysferlin-deficient muscular dystrophy. Nature 423, 168-172, doi:10.1038/nature01573 (2003).

$5 \quad$ Sher, R. B. et al. A rostrocaudal muscular dystrophy caused by a defect in choline kinase beta, the first enzyme in phosphatidylcholine biosynthesis. J Biol Chem 281, 4938-4948, doi:10.1074/jbc.M512578200 (2006).

6 Mitsuhashi, S. et al. Muscle choline kinase beta defect causes mitochondrial dysfunction and increased mitophagy. Hum Mol Genet 20, 3841-3851, doi:10.1093/hmg/ddr305 (2011).

7 Wu, G., Sher, R. B., Cox, G. A. \& Vance, D. E. Understanding the muscular dystrophy caused by deletion of choline kinase beta in mice. Biochim Biophys Acta 1791, 347-356, doi:10.1016/j.bbalip.2009.02.006 (2009).

8 Mitsuhashi, S. et al. A congenital muscular dystrophy with mitochondrial structural abnormalities caused by defective de novo phosphatidylcholine biosynthesis. Am J Hum Genet 88, 845-851, doi:10.1016/j.ajhg.2011.05.010 (2011).

9 Gutierrez Rios, P. et al. Congenital megaconial myopathy due to a novel defect in the choline kinase Beta gene. Arch Neurol 69, 657-661, doi:10.1001/archneurol.2011.2333 (2012).

10 Quinlivan, R. et al. Muscular dystrophy with large mitochondria associated with mutations in the CHKB gene in three British patients: extending the clinical and pathological phenotype. Neuromuscul Disord 23, 549-556, doi:10.1016/j.nmd.2013.04.002 (2013).

11 Castro-Gago, M. et al. Exome sequencing identifies a CHKB mutation in Spanish patient with megaconial congenital muscular dystrophy and mtDNA depletion. Eur J Paediatr Neurol 18, 796-800, doi:10.1016/j.ejpn.2014.06.005 (2014).

12 Cabrera-Serrano, M. et al. Novel CHKB mutation expands the megaconial muscular dystrophy phenotype. Muscle Nerve 51, 140-143, doi:10.1002/mus.24446 (2015).

13 Oliveira, J. et al. New splicing mutation in the choline kinase beta (CHKB) gene causing a muscular dystrophy detected by whole-exome sequencing. J Hum Genet 60, 305-312, doi:10.1038/jhg.2015.20 (2015).

14 Chan, S. H. et al. Megaconial congenital muscular dystrophy: Same novel homozygous mutation in CHKB gene in two unrelated Chinese patients. Neuromuscul Disord 30, 4753, doi:10.1016/j.nmd.2019.10.009 (2020).

15 Lovering, R. M., Porter, N. C. \& Bloch, R. J. The muscular dystrophies: from genes to therapies. Phys Ther 85, 1372-1388 (2005).

16 Ervasti, J. M., Ohlendieck, K., Kahl, S. D., Gaver, M. G. \& Campbell, K. P. Deficiency of a glycoprotein component of the dystrophin complex in dystrophic muscle. Nature 345, 315-319, doi:10.1038/345315a0 (1990). 
17 Michele, D. E. \& Campbell, K. P. Dystrophin-glycoprotein complex: post-translational processing and dystroglycan function. J Biol Chem 278, 15457-15460, doi:10.1074/jbc.R200031200 (2003).

18 Cohn, R. D. \& Campbell, K. P. Molecular basis of muscular dystrophies. Muscle Nerve 23, 1456-1471 (2000).

19 Gawlik, K. I. At the Crossroads of Clinical and Preclinical Research for Muscular Dystrophy-Are We Closer to Effective Treatment for Patients? Int J Mol Sci 19, doi:10.3390/ijms19051490 (2018).

20 Hoffman, E. P. et al. Novel approaches to corticosteroid treatment in Duchenne muscular dystrophy. Phys Med Rehabil Clin N Am 23, 821-828, doi:10.1016/j.pmr.2012.08.003 (2012).

21 Smith, I. C., Bombardier, E., Vigna, C. \& Tupling, A. R. ATP consumption by sarcoplasmic reticulum $\mathrm{Ca}(2)(+)$ pumps accounts for $40-50 \%$ of resting metabolic rate in mouse fast and slow twitch skeletal muscle. PLoS One 8, e68924, doi:10.1371/journal.pone.0068924 (2013).

22 Casares, D., Escriba, P. V. \& Rossello, C. A. Membrane Lipid Composition: Effect on Membrane and Organelle Structure, Function and Compartmentalization and Therapeutic Avenues. Int J Mol Sci 20, doi:10.3390/ijms20092167 (2019).

23 Gervois, P., Torra, I. P., Fruchart, J. C. \& Staels, B. Regulation of lipid and lipoprotein metabolism by PPAR activators. Clin Chem Lab Med 38, 3-11, doi:10.1515/CCLM.2000.002 (2000).

24 Kular, J. et al. Choline kinase beta mutant mice exhibit reduced phosphocholine, elevated osteoclast activity, and low bone mass. J Biol Chem 290, 1729-1742, doi:10.1074/jbc.M114.567966 (2015).

25 Weibel, E. R. et al. Design of the mammalian respiratory system. IV Morphometric estimation of pulmonary diffusing capacity; critical evaluation of new sampling method. Respir Physiol 44, 39-59, doi:10.1016/0034-5687(81)90076-1 (1981).

26 Torres-Palsa, M. J. et al. Expression of intercellular adhesion molecule-1 by myofibers in mdx mice. Muscle Nerve 52, 795-802, doi:10.1002/mus.24626 (2015).

27 Phua, W. W. T., Wong, M. X. Y., Liao, Z. \& Tan, N. S. An aPPARent Functional Consequence in Skeletal Muscle Physiology via Peroxisome Proliferator-Activated Receptors. Int J Mol Sci 19, doi:10.3390/ijms19051425 (2018).

28 Varga, T., Czimmerer, Z. \& Nagy, L. PPARs are a unique set of fatty acid regulated transcription factors controlling both lipid metabolism and inflammation. Biochim Biophys Acta 1812, 1007-1022, doi:10.1016/j.bbadis.2011.02.014 (2011).

29 Viswakarma, N. et al. Coactivators in PPAR-Regulated Gene Expression. PPAR Res 2010, doi:10.1155/2010/250126 (2010).

30 Mitsuhashi, S. \& Nishino, I. Phospholipid synthetic defect and mitophagy in muscle disease. Autophagy 7, 1559-1561, doi:10.4161/auto.7.12.17925 (2011).

31 Caviglia, J. M., De Gomez Dumm, I. N., Coleman, R. A. \& Igal, R. A. Phosphatidylcholine deficiency upregulates enzymes of triacylglycerol metabolism in CHO cells. J Lipid Res 45, 1500-1509, doi:10.1194/j1r.M400079-JLR200 (2004).

32 Jacobs, R. L., Devlin, C., Tabas, I. \& Vance, D. E. Targeted deletion of hepatic CTP:phosphocholine cytidylyltransferase alpha in mice decreases plasma high density and very low density lipoproteins. J Biol Chem 279, 47402-47410, doi:10.1074/jbc.M404027200 (2004). 
33 Jackowski, S., Wang, J. \& Baburina, I. Activity of the phosphatidylcholine biosynthetic pathway modulates the distribution of fatty acids into glycerolipids in proliferating cells. Biochim Biophys Acta 1483, 301-315, doi:10.1016/s1388-1981(99)00203-6 (2000).

34 Benador, I. Y., Veliova, M., Liesa, M. \& Shirihai, O. S. Mitochondria Bound to Lipid Droplets: Where Mitochondrial Dynamics Regulate Lipid Storage and Utilization. Cell Metab 29, 827-835, doi:10.1016/j.cmet.2019.02.011 (2019).

35 Sayed-Zahid, A. A. et al. Functional rescue in a mouse model of congenital muscular dystrophy with megaconial myopathy. Hum Mol Genet 28, 2635-2647, doi:10.1093/hmg/ddz068 (2019).

36 Poulsen, L., Siersbaek, M. \& Mandrup, S. PPARs: fatty acid sensors controlling metabolism. Semin Cell Dev Biol 23, 631-639, doi:10.1016/j.semcdb.2012.01.003 (2012).

37 Wang, Y. X. et al. Peroxisome-proliferator-activated receptor delta activates fat metabolism to prevent obesity. Cell 113, 159-170, doi:10.1016/s0092-8674(03)00269-1 (2003).

38 Finck, B. N. et al. The cardiac phenotype induced by PPARalpha overexpression mimics that caused by diabetes mellitus. $J$ Clin Invest 109, 121-130, doi:10.1172/JCI14080 (2002).

39 Finck, B. N. et al. A potential link between muscle peroxisome proliferator- activated receptor-alpha signaling and obesity-related diabetes. Cell Metab 1, 133-144, doi:10.1016/j.cmet.2005.01.006 (2005).

40 Capogrosso, R. F. et al. Ryanodine channel complex stabilizer compound S48168/ARM210 as a disease modifier in dystrophin-deficient mdx mice: proof-ofconcept study and independent validation of efficacy. FASEB J 32, 1025-1043, doi:10.1096/fj.201700182RRR (2018).

41 Shahini, A. et al. Efficient and high yield isolation of myoblasts from skeletal muscle. Stem Cell Res 30, 122-129, doi:10.1016/j.scr.2018.05.017 (2018).

42 Rayavarapu, S. et al. Characterization of dysferlin deficient SJL/J mice to assess preclinical drug efficacy: fasudil exacerbates muscle disease phenotype. PLoS One $\mathbf{5}$, e12981, doi:10.1371/journal.pone.0012981 (2010).

43 Quinn, J. L. et al. Effects of Dantrolene Therapy on Disease Phenotype in Dystrophin Deficient mdx Mice. PLoS Curr 5, doi:10.1371/currents.md.e246cf493a7edb1669f42fb735936b46 (2013).

44 Bligh, E. G. \& Dyer, W. J. A rapid method of total lipid extraction and purification. Can J Biochem Physiol 37, 911-917, doi:10.1139/o59-099 (1959).

45 McCloy, R. A. et al. Partial inhibition of Cdk1 in G 2 phase overrides the SAC and decouples mitotic events. Cell Cycle 13, 1400-1412, doi:10.4161/cc.28401 (2014).

46 Weibel, E. R. Stereological methods in cell biology: where are we--where are we going? J Histochem Cytochem 29, 1043-1052, doi:10.1177/29.9.7026667 (1981).

47 Meinild Lundby, A. K. et al. Exercise training increases skeletal muscle mitochondrial volume density by enlargement of existing mitochondria and not de novo biogenesis. Acta Physiol (Oxf) 222, doi:10.1111/apha.12905 (2018). 


\section{FIGURES}

Fig. 1

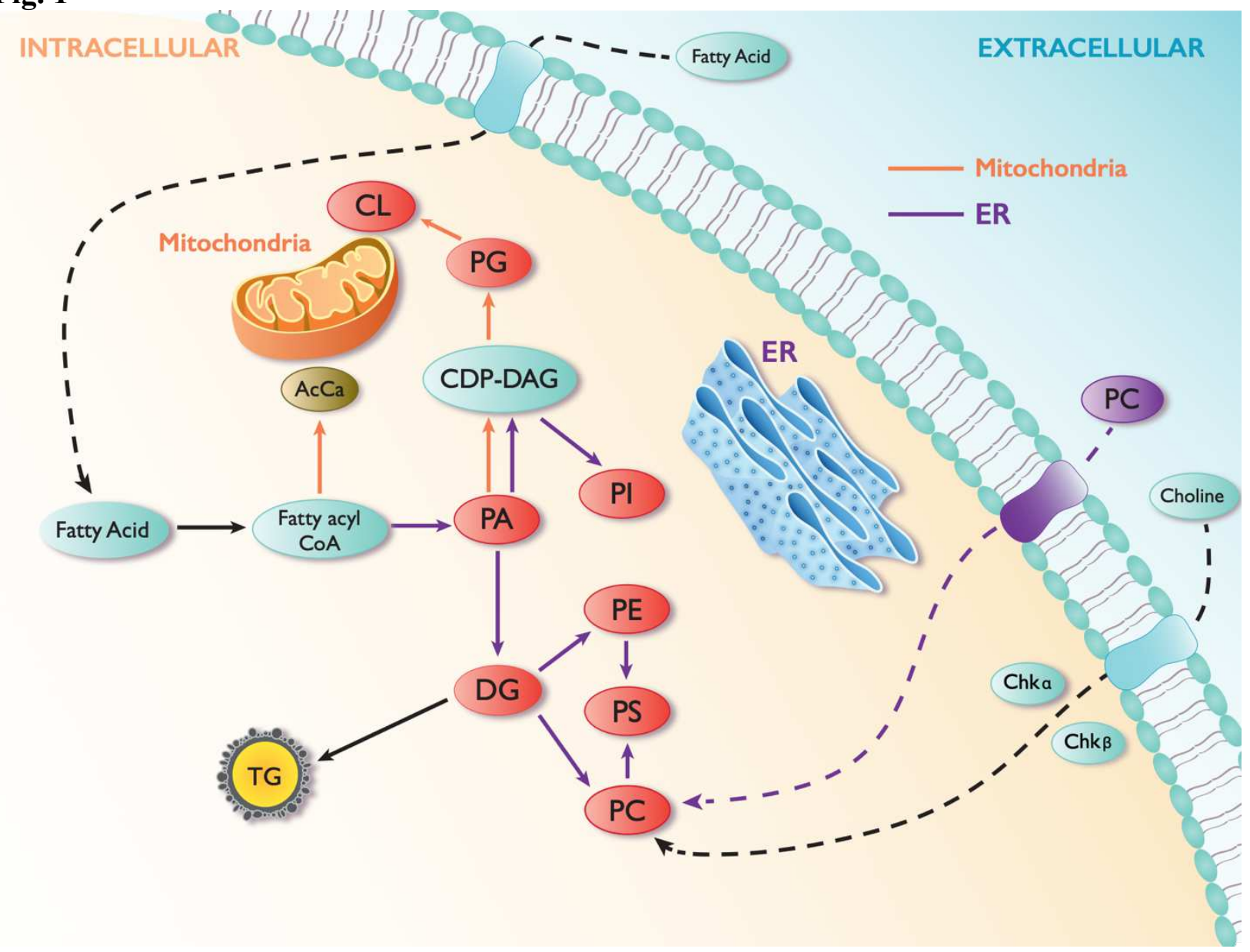


Fig. 2
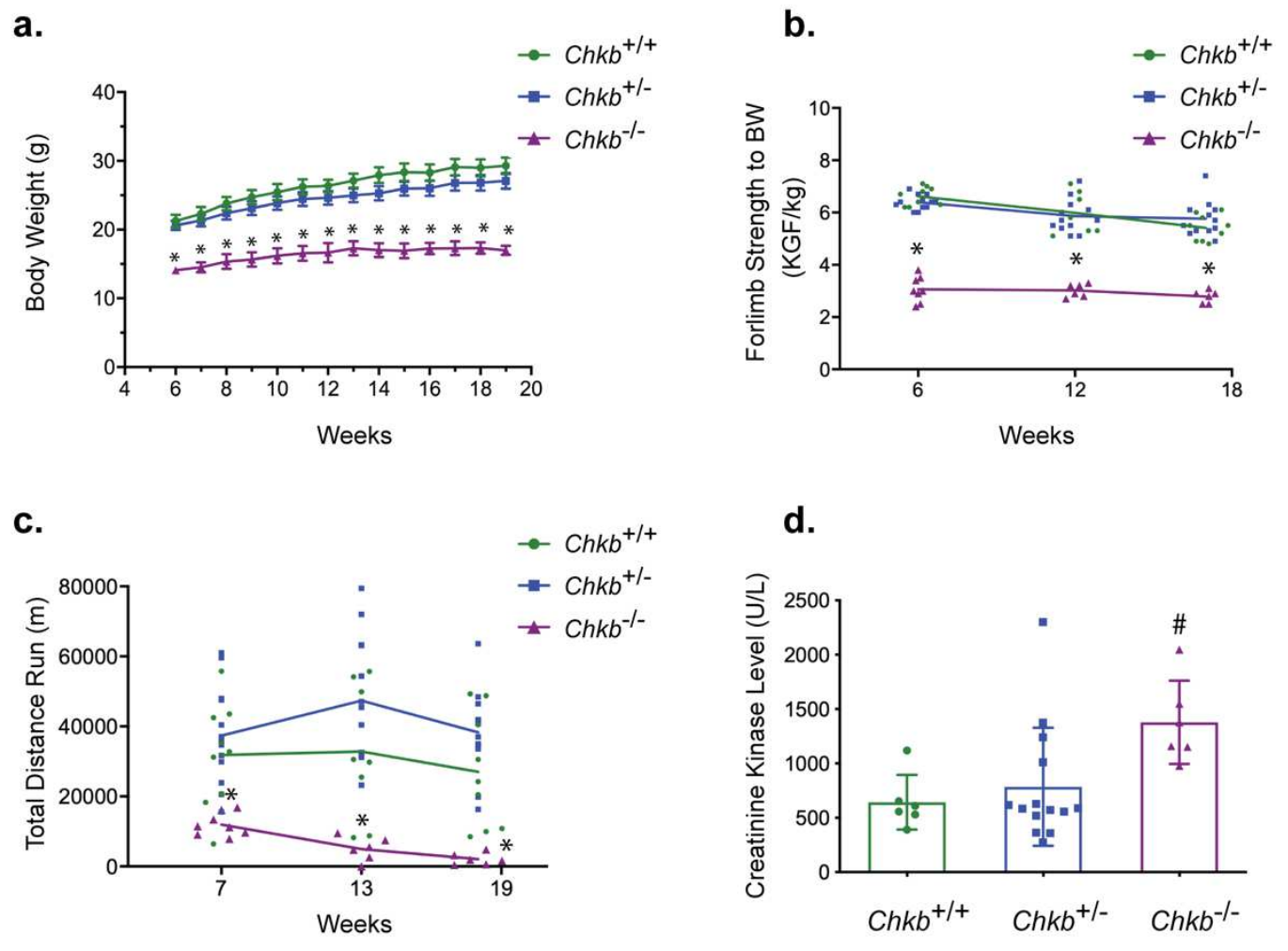

d.

e.

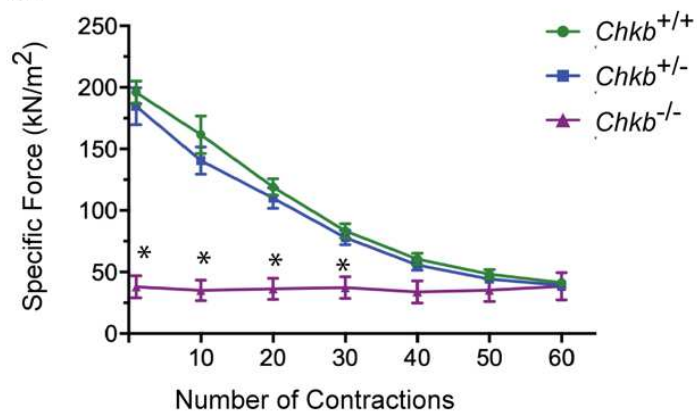

f.

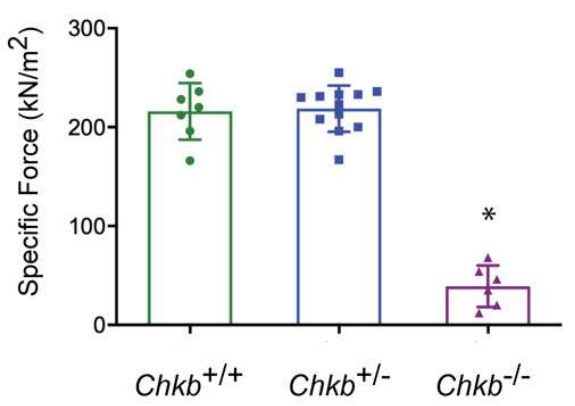


Fig. 3

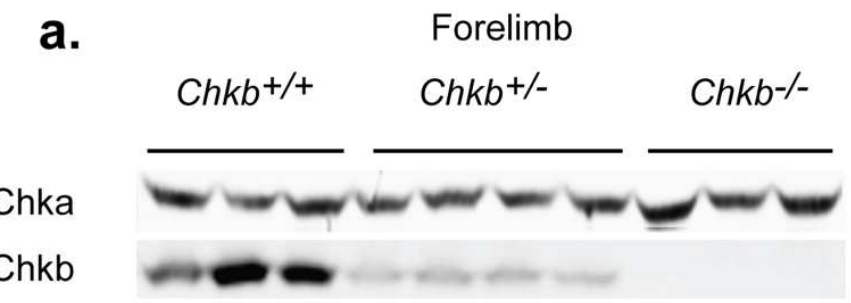

Gapdh
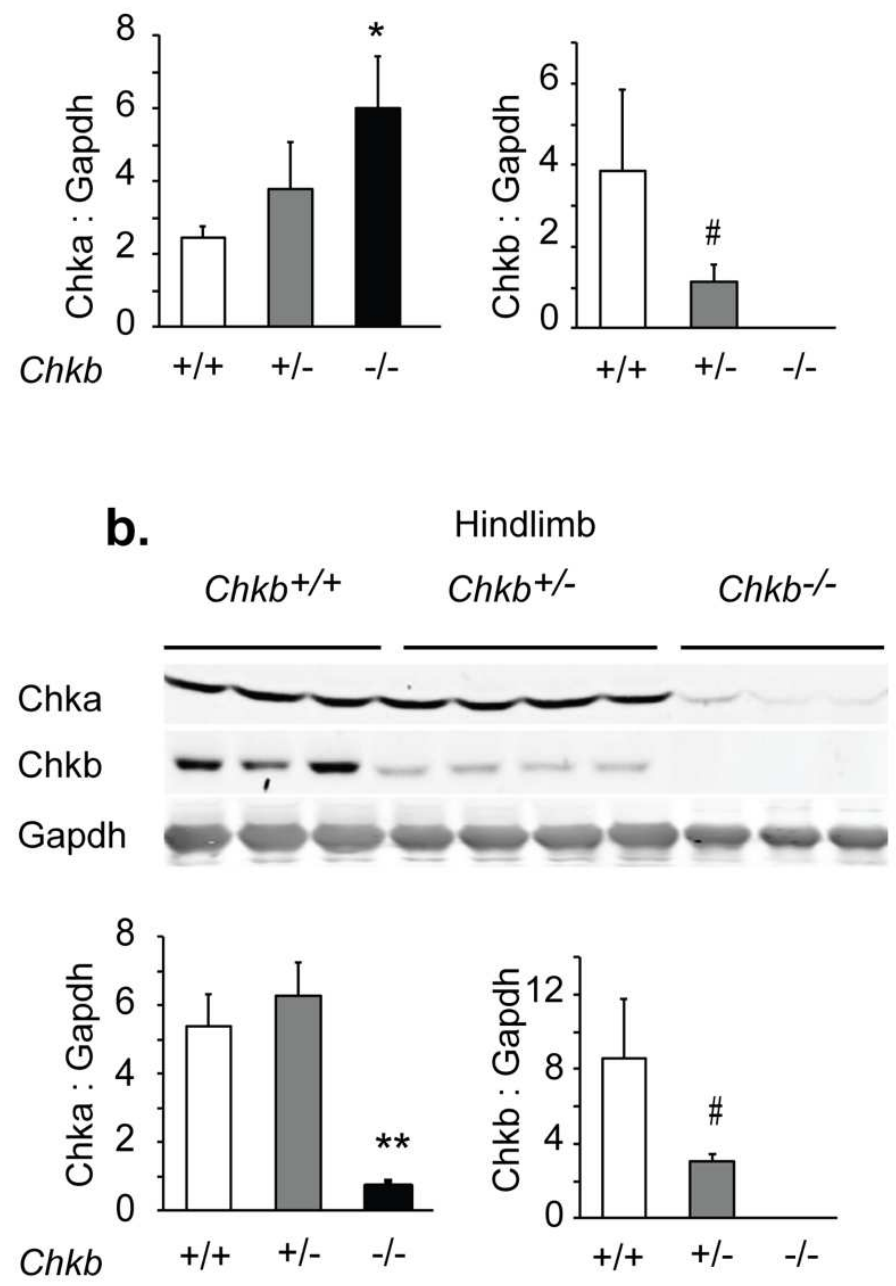


\section{Fig. 4}
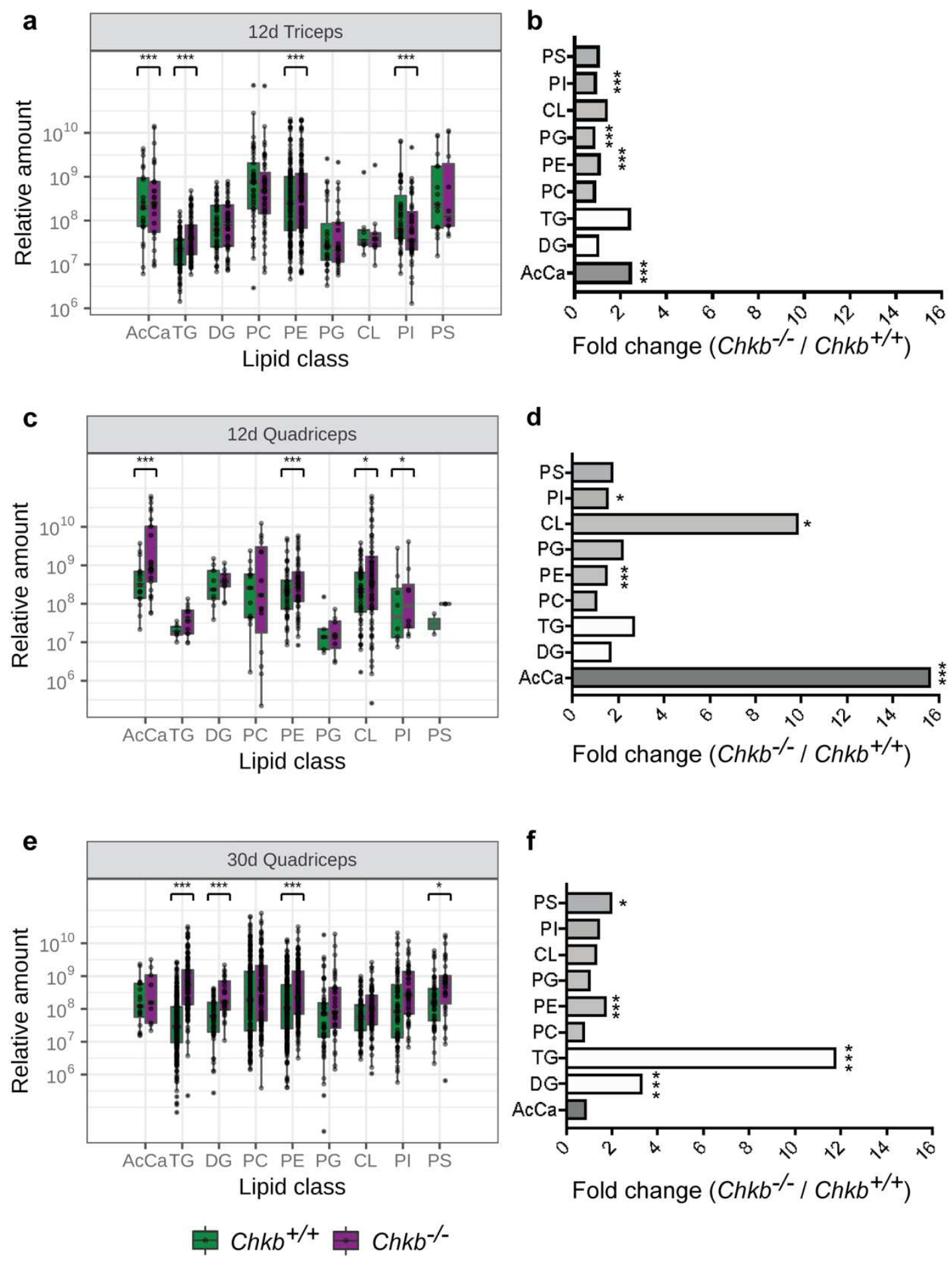
Fig. 5

a.
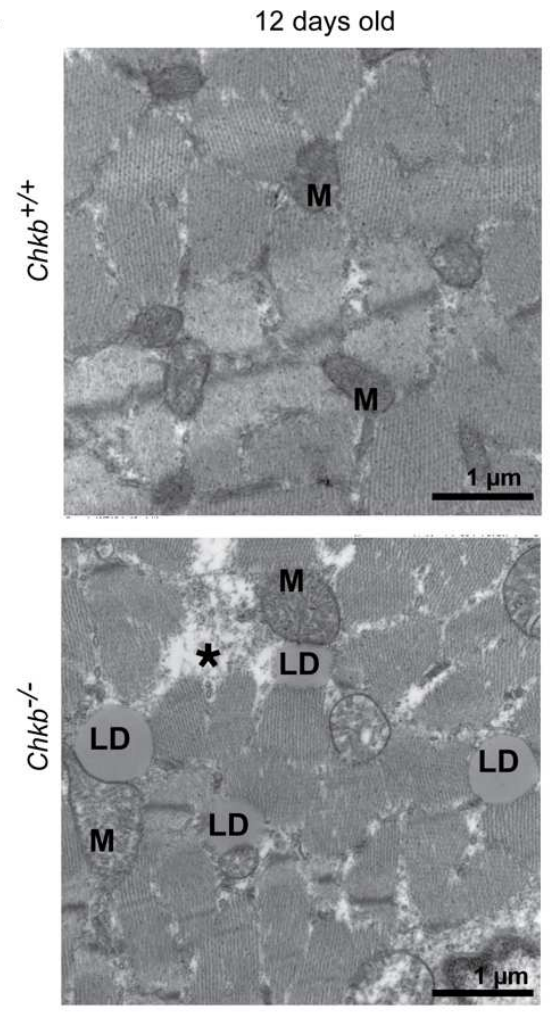

b.

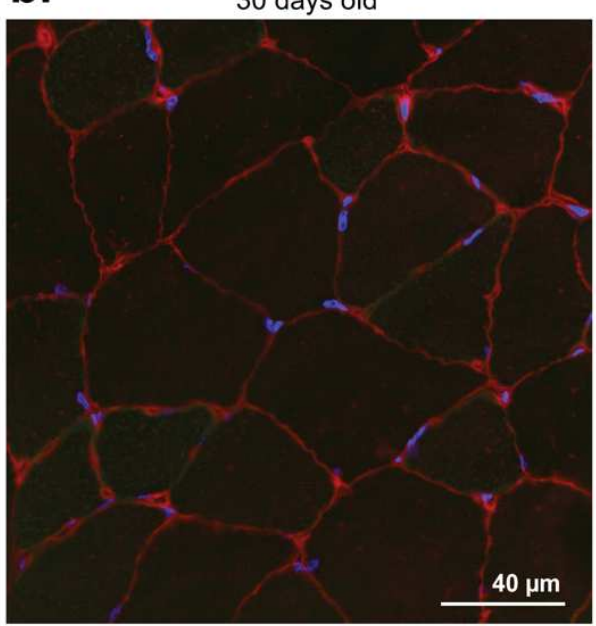

115 days old
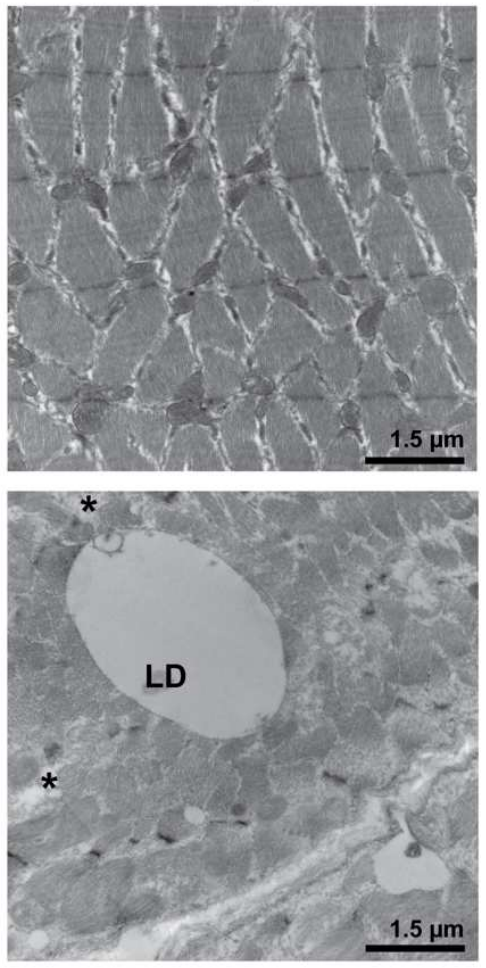

$\mathrm{Chkb}^{-/-}$

30 days old

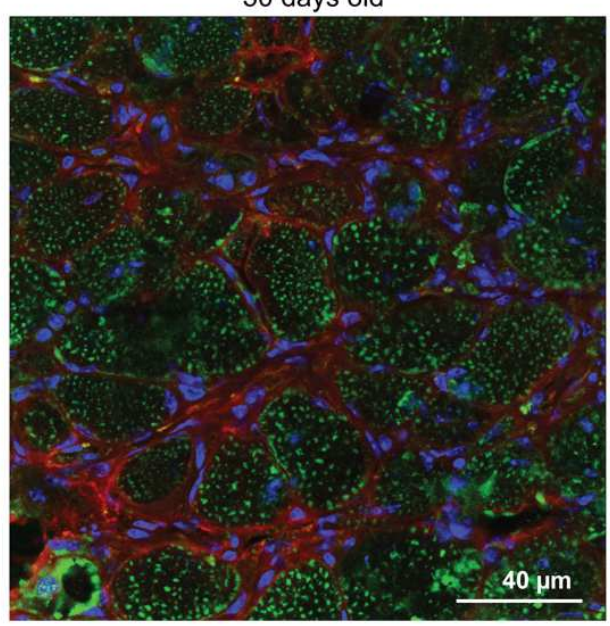


Fig. 6

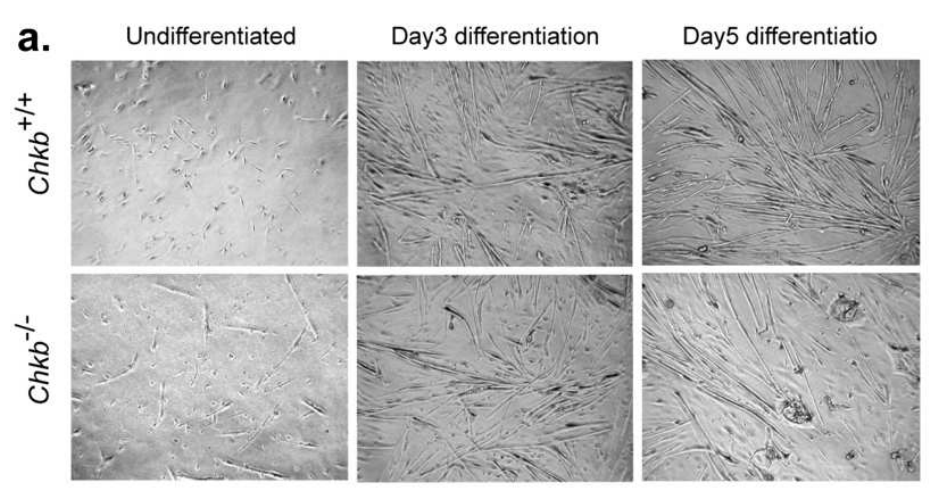

b.
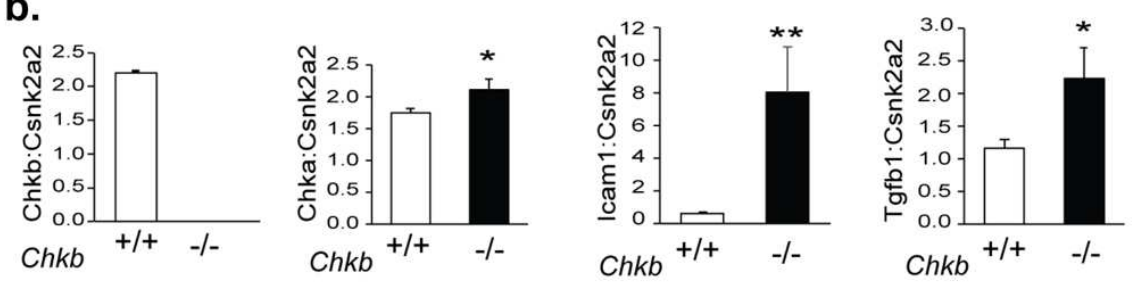

c.

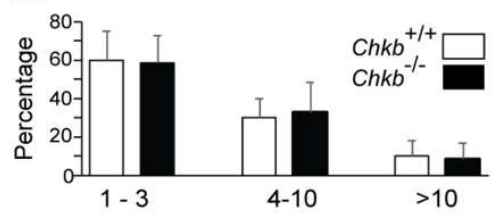

d. Nuclei in myotube/

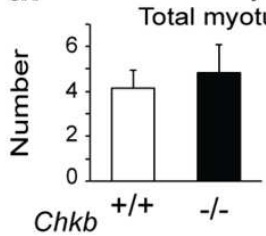

e.

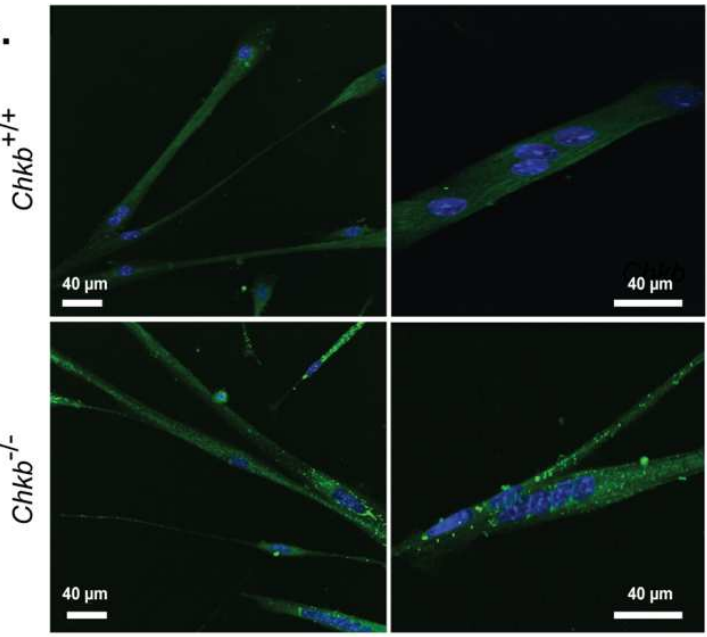

f.

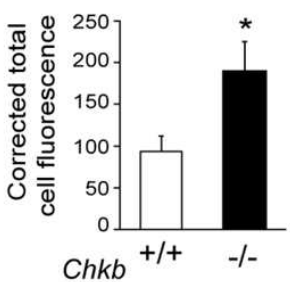


Fig. 7
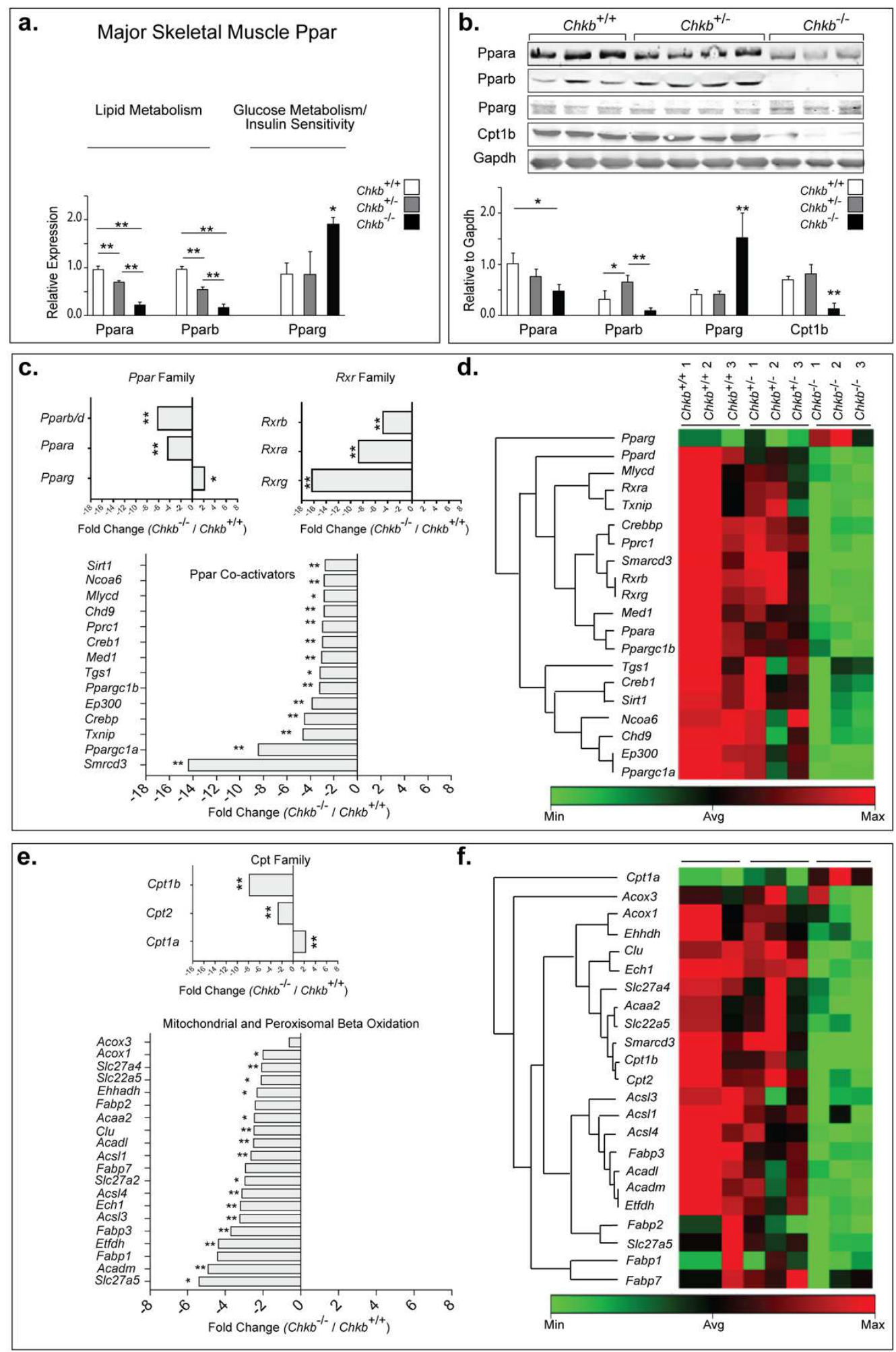

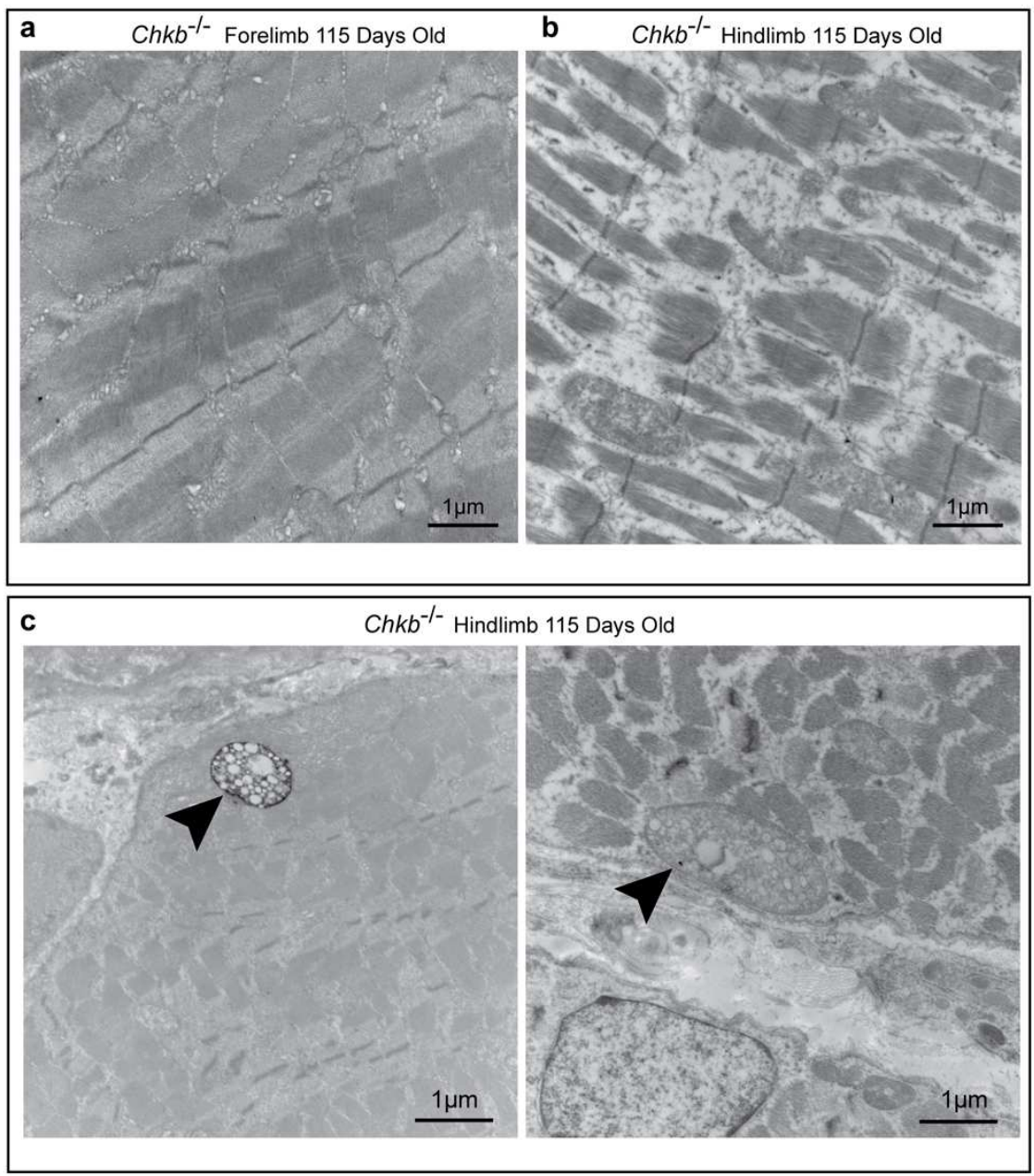

\section{Supplementary Fig. 1| Ultrastructural abnormalities in skeletal muscles of Chkb defi-} cient mice.

a and $\mathbf{b}$. Transmission electron microscopy (TEM) appearance of the forelimb (Triceps) (a) and hindlimb (Quadriceps) (b) of 115 days old $\mathrm{Chkb}^{-l-}$ mice showing extensive injury in hindlimb not the forelimb. c, Ultrastructural appearance of lipofuscin granules (arrowheads), heterogeneous in their size and structure observed in gastrocnemius and quadriceps samples from 115 days old Chkb deficient 
a
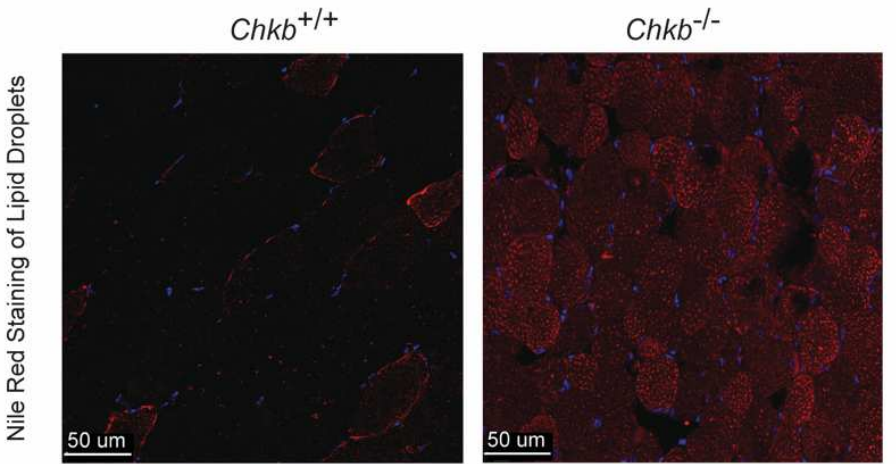

b
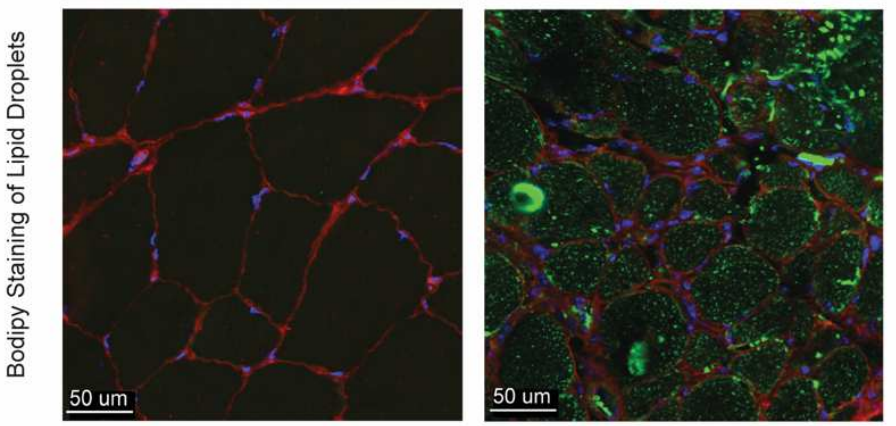

c

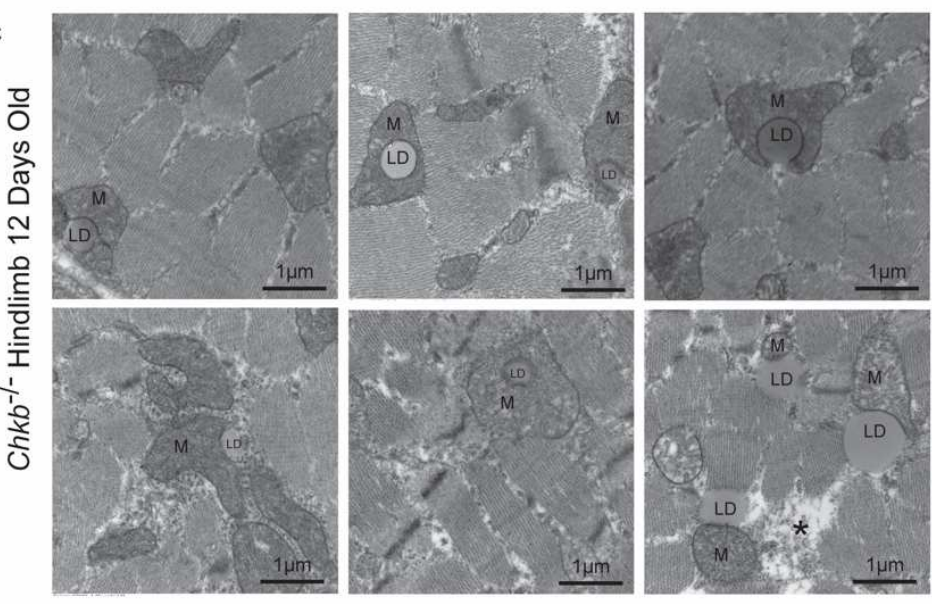

Supplementary Fig. 2| Increased intramyocellular lipid droplet accumulation in hindlimb

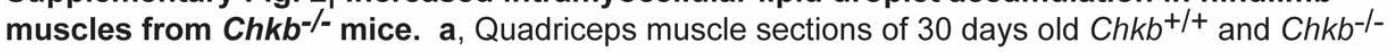
mice were fixed and stained with Nile Red-550 / $640 \mathrm{~nm}$ to visualize LDs (Red). Dapi was used to stain nucleus (Blue). (representative of 3 mice per group). b, Quadriceps muscle sections of 30 days old $\mathrm{Chkb}^{+/+}$and $\mathrm{Chkb}^{-/-}$mice were fixed and stained with BODIPY-493/503 to visualize LDs (Green). Concanavalin A dye conjugate (CF TM 633) and Dapi were used to stain membrane (Red) and nucleus (Blue) respectively. (representative of 3 mice per group).c, Transmission electron microscopy (TEM) appearance of the hindlimb muscle samples of 12 days old $\mathrm{Chkb}^{-/}$- mice showing the high prevalence of peri-droplet mitochondria in hindlimb muscles (representative of 3 mice per group). Scale bar $1 \mu \mathrm{m}$. LD=Lipid droplets. $M=$ Mitochondria. *=Disrupted sarcomeres. 
a.

12 days old
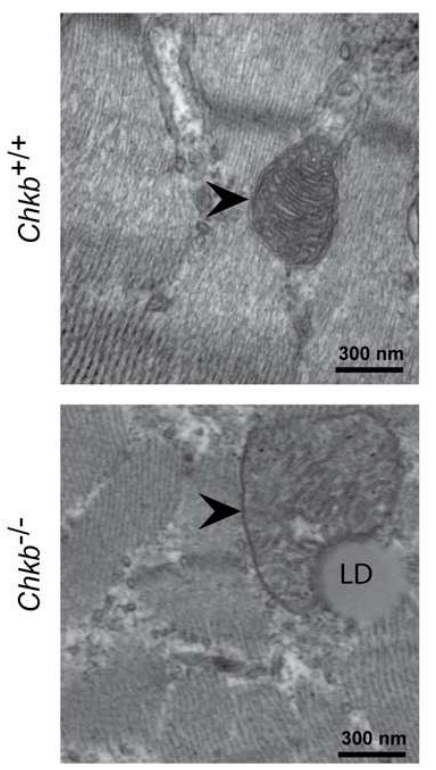

60 days old
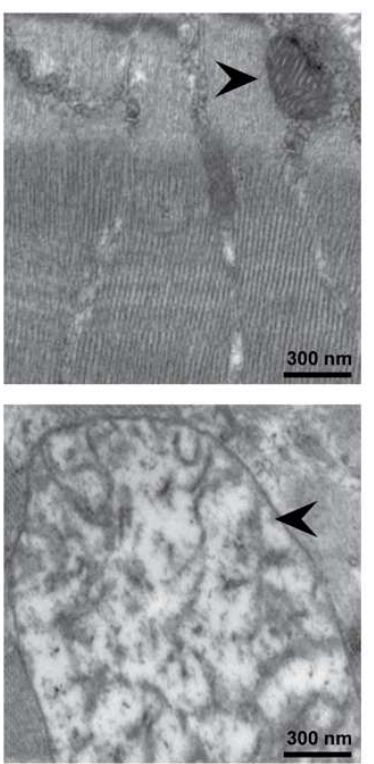

b.
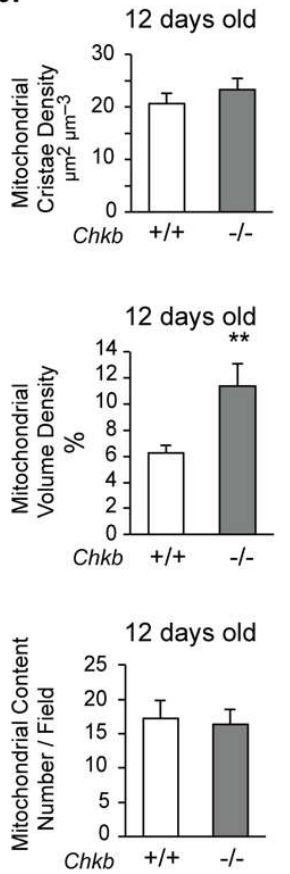

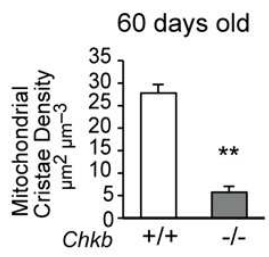

60 days old
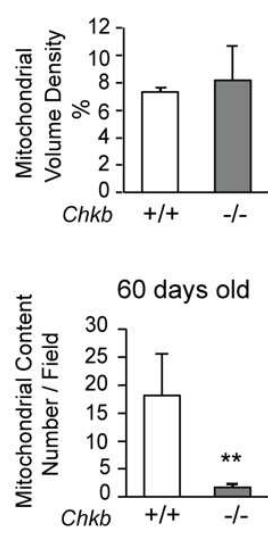

Supplementary Fig.3 | Mitochondrial profile quantification. a, transmission electron microscopy (TEM) appearance of the mitochondrial profile of hindlimbs from 12 days old and 60 days old wild-type $\left(\mathrm{Chkb}^{+/+}\right)$and Chkb-deficient (Chkb-/-) mice (representative of 3 mice per group). b, At 12 days of age hindlimbs from wild type and $\mathrm{Chkb}^{-/-}$mice had the same number of mitochondria per imaged field however, the volume density of the $\mathrm{Chkb}^{-/-}$mitochondria was

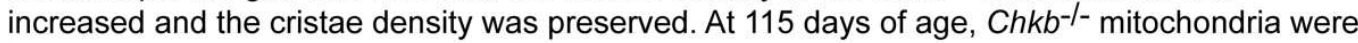
fewer in number, and had markedly reduced cristae density and were much larger in size. The increased size of the mitochondria at this age accounts for the preserved volume density. 
a.

Forelimb

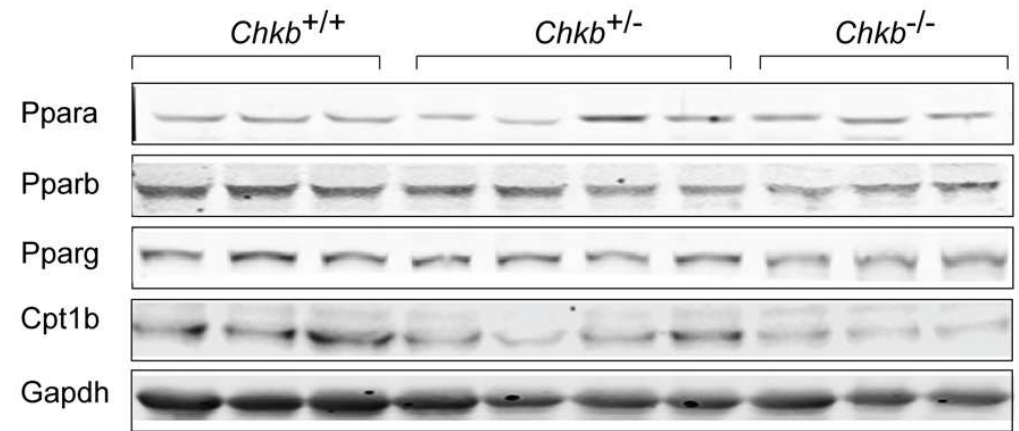

b.
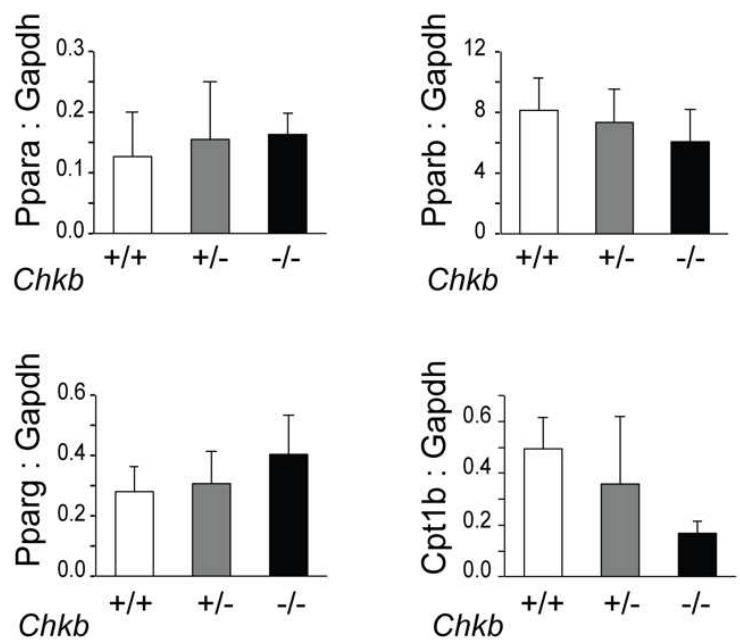

Supplementary Fig. 4| Protein expression of the members of the Ppar family in forelimb samples. a, Western blot of forelimb (Triceps) samples from three distinct (lanes 1-3) $\mathrm{Chkb}^{+/+}$, four distinct (lanes 4-7) $\mathrm{Chkb}^{+/-}$and three distinct (lanes 8-10) $\mathrm{Chkb}^{-/-}$mice probed with anti-Ppara, anti- Pparb, anti-Pparg, anti- Cpt1b and anti-Gapdh antibodies. b, densitometry of the WB data shows the ratio of Ppara, Pparb, Pparg and Cpt1b to Gapdh.Values are means \pm SD; $n=3-4$ per group. No significant difference was obserd among groups using one-way ANOVA with Tukey's multiple comparison test. 


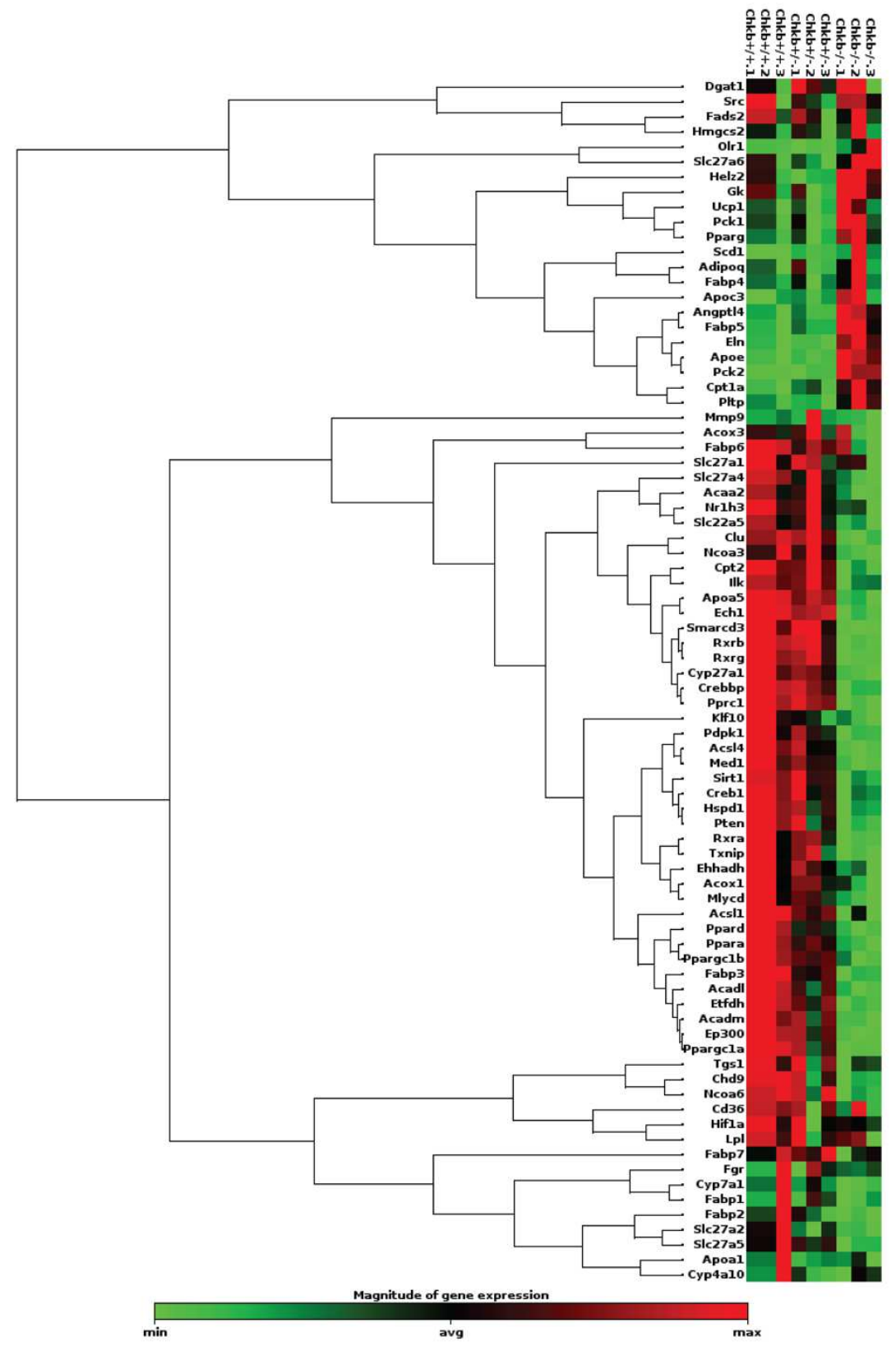

Supplementary Fig.5 | Chkb regulates the expression of the members of the Ppar family as well as Ppar target genes. Clustergram showing non-supervised hierarchical clustering of the entire dataset to display a heat map with dendrograms indicating co-regulated genes across groups or individual samples. Sample Dimension: 1D. Join Type: Average. Color Coded: Average Genes. 


\section{Supplementary Table 1| Ppar associated genes under-expressed in Chkb ${ }^{-/}$ hindlimb vs. Chkb ${ }^{+/+}$hindlimb.}

\begin{tabular}{|c|c|c|c|}
\hline $\begin{array}{l}\text { Gene } \\
\text { Symbol }\end{array}$ & $\begin{array}{l}\text { Fold } \\
\text { Regulation }\end{array}$ & p-Value & Description \\
\hline Rxrg & -16.38 & 0.000250 & Retinoid $\mathrm{X}$ receptor gamma \\
\hline Smarcd3 & -14.40 & 0.000951 & $\begin{array}{l}\text { SWI/SNF related, matrix associated, actin dependent regulator of chromatin, subfamily } \mathrm{d} \text {, } \\
\text { member } 3\end{array}$ \\
\hline Rxra & -8.78 & 0.008824 & Retinoid X receptor alpha \\
\hline Ppargc1a & -8.46 & 0.000002 & Peroxisome proliferative activated receptor, gamma, coactivator 1 alpha \\
\hline Cpt1b & -7.93 & 0.002692 & Carnitine palmitoyltransferase $1 \mathrm{~b}$, muscle \\
\hline Pparb & -6.19 & 0.000176 & Peroxisome proliferator activator receptor delta \\
\hline Cyp27a1 & -5.58 & 0.001538 & Cytochrome P450, family 27 , subfamily a, polypeptide 1 \\
\hline S/c27a5 & -5.43 & 0.022345 & Solute carrier family 27 (fatty acid transporter), member 5 \\
\hline Hspd1 & -5.05 & 0.001198 & Heat shock protein 1 (chaperonin) \\
\hline Acadm & -4.95 & 0.000659 & Acyl-Coenzyme A dehydrogenase, medium chain \\
\hline Rxrb & -4.80 & 0.000043 & Retinoid X receptor beta \\
\hline Txnip & -4.68 & 0.008384 & Thioredoxin interacting protein \\
\hline Crebbp & -4.54 & 0.000152 & CREB binding protein \\
\hline Ppara & -4.42 & 0.000517 & Peroxisome proliferator activated receptor alpha \\
\hline Etfdh & -4.39 & 0.000073 & Electron transferring flavoprotein, dehydrogenase \\
\hline Apoa5 & -4.07 & 0.000064 & Apolipoprotein A-V \\
\hline Ep300 & -3.89 & 0.000062 & E1A binding protein $\mathrm{p} 300$ \\
\hline Fabp3 & -3.75 & 0.000022 & Fatty acid binding protein 3, muscle and heart \\
\hline Acs/3 & -3.33 & 0.000350 & Acyl-CoA synthetase long-chain family member 3 \\
\hline Ppargc1b & -3.25 & 0.001233 & Peroxisome proliferative activated receptor, gamma, coactivator 1 beta \\
\hline Ech1 & -3.24 & 0.000023 & Enoyl coenzyme A hydratase 1, peroxisomal \\
\hline Tgs1 & -3.23 & 0.028174 & Trimethylguanosine synthase \\
\hline Acs/4 & -3.15 & 0.000615 & Acyl-CoA synthetase long-chain family member 4 \\
\hline Med1 & -3.10 & 0.001997 & Mediator complex subunit 1 \\
\hline Pdpk1 & -3.08 & 0.008230 & 3-phosphoinositide dependent protein kinase 1 \\
\hline Creb1 & -3.02 & 0.003162 & CAMP responsive element binding protein 1 \\
\hline Pprc1 & -3.02 & 0.000136 & Peroxisome proliferative activated receptor, gamma, coactivator-related 1 \\
\hline Slc27a2 & -2.98 & 0.017603 & Solute carrier family 27 (fatty acid transporter), member 2 \\
\hline Chd9 & -2.89 & 0.000078 & Chromodomain helicase DNA binding protein 9 \\
\hline Mlycd & -2.89 & 0.010148 & Malonyl-CoA decarboxylase \\
\hline Ncoa6 & -2.86 & 0.000177 & Nuclear receptor coactivator 6 \\
\hline Sirt1 & -2.79 & 0.001001 & Sirtuin 1 (silent mating type information regulation 2, homolog) 1 \\
\hline Cpt2 & -2.77 & 0.002056 & Carnitine palmitoyltransferase 2 \\
\hline Acs/1 & -2.67 & 0.006296 & Acyl-CoA synthetase long-chain family member 1 \\
\hline Acadl & -2.54 & 0.000127 & Acyl-Coenzyme A dehydrogenase, long-chain \\
\hline Clu & -2.52 & 0.000146 & Clusterin \\
\hline Acaa2 & -2.48 & 0.010777 & Acetyl-Coenzyme A acyltransferase 2 (mitochondrial 3-oxoacyl-Coenzyme A thiolase) \\
\hline KIf10 & -2.42 & 0.009957 & Kruppel-like factor 10 \\
\hline Ehhadh & -2.34 & 0.022919 & Enoyl-Coenzyme A, hydratase/3-hydroxyacyl Coenzyme A dehydrogenase \\
\hline Pten & -2.30 & 0.000522 & Phosphatase and tensin homolog \\
\hline Slc22a5 & -2.13 & 0.010148 & Solute carrier family 22 (organic cation transporter), member 5 \\
\hline Slc27a4 & -2.11 & 0.001068 & Solute carrier family 27 (fatty acid transporter), member 4 \\
\hline Acox1 & -2.04 & 0.034786 & Acyl-Coenzyme A oxidase 1, palmitoyl \\
\hline Nr1h3 & -2.02 & 0.020303 & Nuclear receptor subfamily 1 , group $\mathrm{H}$, member 3 \\
\hline
\end{tabular}


Fold Regulation cut off $=2$. $p-$ Value cut off $=0.05$. Fold-Change $\left(2^{\wedge}(-\right.$ Delta Delta CT $\left.)\right)$ is the normalized gene expression $\left(2^{\wedge}(-\right.$ Delta CT $\left.)\right)$ in the Test Sample divided the normalized gene expression $\left(2^{\wedge}\right.$ (- Delta CT) ) in the Control Sample. Fold-change values less than one indicate a negative or down-regulation, and the fold-regulation is the negative inverse of the fold-change. The $p$ values are calculated based on a Student's ttest of the replicate $2^{\wedge}$ (- Delta CT) values for each gene in the control group and Chkb deficient groups. 


\section{Supplementary Table 2 | Ppar associated genes over-expressed in Chkb-/- hindlimb vs. Chkb ${ }^{+/+}$hindlimb}

\begin{tabular}{|l|l|l|l|}
\hline $\begin{array}{l}\text { Gene } \\
\text { Symbol }\end{array}$ & $\begin{array}{l}\text { Fold } \\
\text { Regulation }\end{array}$ & $p$-Value & Description \\
\hline Pck2 & 8.55 & 0.000216 & Phosphoenolpyruvate carboxykinase 2 (mitochondrial) \\
\hline Apoe & 6.77 & 0.000851 & Apolipoprotein E \\
\hline Angptl4 & 4.46 & 0.007248 & Angiopoietin-like 4 \\
\hline Eln & 3.46 & 0.002955 & Elastin \\
\hline Fabp5 & 2.74 & 0.009434 & Fatty acid binding protein 5, epidermal \\
\hline Pltp & 2.61 & 0.030470 & Phospholipid transfer protein \\
\hline Cpt1a & 2.29 & 0.008722 & Carnitine palmitoyltransferase 1a, liver \\
\hline Pparg & 2.24 & 0.041818 & Peroxisome proliferator activated receptor gamma \\
\hline
\end{tabular}

Fold Regulation cut off $=2$. $p$-Value cut off $=0.05$. Fold-Change $\left(2^{\wedge}(-\right.$ Delta Delta CT $\left.)\right)$ is the normalized gene expression $\left(2^{\wedge}(-\right.$ Delta CT $\left.)\right)$ in the Test Sample divided the normalized gene expression $\left(2^{\wedge}\right.$ (- Delta CT)) in the Control Sample. Fold-change values more than one indicate a positive or up-regulation. The $p$ values are calculated based on a Student's t-test of the replicate $2^{\wedge}$ (- Delta CT) values for each gene in the control group and Chkb deficient groups. 